AEI-2009-013

\title{
The One-loop Open Superstring Massless Five-point Amplitude with the Non-Minimal Pure Spinor Formalism
}

\author{
Carlos R. Mafrat and Christian Stahn ${ }^{2}$ \\ ${ }^{1}$ Max-Planck-Institut für Gravitationsphysik, Albert-Einstein-Institut \\ 14476 Golm, Germany \\ ${ }^{2}$ Department of Physics and Astronomy, University of Rochester \\ Rochester, NY 14627-0171, USA
}

\begin{abstract}
We compute the massless five-point amplitude of open superstrings using the nonminimal pure spinor formalism and obtain a simple kinematic factor in pure spinor superspace, which can be viewed as the natural extension of the kinematic factor of the massless four-point amplitude. It encodes bosonic and fermionic external states in supersymmetric form and reduces to existing bosonic amplitudes when expanded in components, therefore proving their equivalence. We also show how to compute the kinematic structures involving fermionic states.
\end{abstract}

February 2009

\footnotetext{
1 email: crmafra@aei.mpg.de

2 email: stahn@pas.rochester.edu
} 


\section{Introduction}

As emphasized by Gross and Sloan [1], superstring scattering amplitudes are useful to derive the low energy effective action of its massless modes. For example, it allows one to obtain stringy corrections to the Yang-Mills and Einstein-Hilbert actions [2].

Up to the year 2000 these computations were done using either the Ramond-NeveuSchwarz (RNS) [3] or Green-Schwarz (GS) [4] formulation in ten-dimensional flat background. In the open (closed) superstring, the tree and one-loop four-gluon (four-graviton) amplitudes lead to an infinite series of higher derivative corrections starting with the well-known $t_{8} F^{4}\left(t_{8} t_{8} R^{4}\right)$ interaction terms [2] [5]. After several years of efforts, the corresponding corrections were finally computed at the two-loop level in a series of papers by D'Hoker and Phong (see [6] and references therein), starting with terms of the form $t_{8} D^{2} F^{4}\left(t_{8} t_{8} D^{4} R^{4}\right)$.

The results above were obtained for the bosonic amplitudes in the NS and NS-NS sectors. While the fermionic four-point amplitudes involving strings in the $\mathrm{R}$ sector are known at tree- and one-loop levels, the two-loop computations using the gauge slice independent methods of [6] are still lacking in the RNS formalism. The problems involving fermionic amplitudes present a major complication to the supersymmetric completion to string effective actions and the analysis of higher-order derivative corrections for RR gauge fields (see e.g. [7]).

This state of affairs, however, started to change with the proposal of the pure spinor formalism [8]. It does not suffer from the inherent difficulties of the RNS and GS formulations responsible for the status quo concerning two-loop fermionic amplitudes. It accomplishes that by having manifest space-time supersymmetry together with a Lorentz invariant BRST quantization procedure 3 .

It was soon realized that tree-level amplitudes computed using this new formalism were equivalent to RNS results [10]. When the multiloop prescription came into existence four years later, it was readily used to obtain a non-renormalization theorem 1 regarding the $R^{4}$ term in the effective action [14] and the two-loop massless four-point amplitude [15] of the type IIB superstring. The two-loop kinematic factor derived in [15] was expressed as

3 The usefulness of the PS formalism is of course not restricted to amplitude calculations, see e.g. the review 9 .

4 This theorem was later extended in [11] (see also [12]) and used by Green, Russo and Vanhove [13] to argue that $N=8$ SUGRA is finite up to 8 loops. 
an integral over pure spinor superspace [16] and was subsequently shown [17] to agree with the two-loop RNS computations of D'Hoker and Phong [6]. Furthermore, explicit bosonic computations also proved the PS/RNS equivalence at one loop [18 and were followed by calculations involving fermionic external states in [19].

In 2008, several manipulations in pure spinor superspace were used to prove that the massless four-point amplitudes at tree-level and at one and two loops are all related to each other [20], being proportional (up to Mandelstam invariants) to the same tree-level kinematic factor. With the identities of [20], the knowledge of all bosonic and fermionic four-point scattering amplitudes up to two loops was obtained altogether simply by possessing the complete tree-level result.

By the end of 2005, Berkovits proposed an extension of the pure spinor formalism by introducing non-minimal variables, which resulted in a simplified expression for the b-ghost [21]. In this non-minimal formalism, the b-ghost is one of the generators of a $\hat{c}=3 N=2$ topological algebra, and it is "simpler" than the corresponding b-ghost of the minimal formalism, albeit still "complicated" due to its composite nature, with poles in $1 /(\lambda \bar{\lambda})$. It was already argued in [21] that multiloop amplitudes computed using the new topological methods should be equivalent to the results computed in the minimal formalism, and that was verified by some explicit amplitude computations in [22]5.

With the non-minimal pure spinor framework being simpler than its version of five years earlier and having a prescription which is in principle valid to all-loop orders [24], a renewed computation of superstring scattering amplitudes is a task worth pursuing and holds the potential to increase our understanding of stringy corrections to low-energy effective actions (see e.g. the treatment of tree-level four-point amplitudes in [25]).

From the inner perspective of the non-minimal pure spinor formalism, the straightforward way with which the expressions for the massless four-point amplitudes were obtained (up to two loops) is related to the fact that in these cases only the zero modes of the b-ghost played a rôle. This allowed the amplitudes in pure spinor superspace to be determined essentially by powerful symmetry arguments [14][15] [22].

The motivation which started this paper was to test the abilities of the pure spinor formalism in a situation where not only the b-ghost zero-modes would (in principle) contribute. In view of the complicated nature of the b-ghost, that motivation was deemed to

\footnotetext{
5 There exists now a formal proof of NMPS/PS equivalence by Hoogeveen and Skenderis [23].
} 
be another non-trivial test whose result was worth knowing. At the same time, the knowledge about higher-point amplitudes involving fermionic external states would benefit in case of a successful outcome. As we will show, the (non-minimal) pure spinor formalism 6 withstands the test and leads to a simple answer in pure spinor superspace.

This paper is organized as follows. In section 2 we recall the prescription of the PS formalism for the computation of the open superstring one-loop amplitude. We propose simplified expressions for the pure spinor integration measures and the normalization factor $\mathcal{N}(y)$ to facilitate explicit computations, allowing one to identify a shortcut which can be taken to integrate out the $w_{\alpha}, \bar{w}^{\alpha}$ and $s^{\alpha}$ variables. In section 3 we prove the vanishing of the contributions coming from the non zero-modes of the b-ghosts and obtain the kinematic factor of this five-point amplitude as a (rather large) expression in pure spinor superspace, relegating the detailed calculations to Appendix B. We then prove its gauge invariance by showing that the gauge variation is a total derivative which vanishes by the cancelled propagator argument. In section 4 we simplify the answer of section 3 by using further pure spinor manipulations to the point of being able to identify a simple form for the massless five-point kinematic factor,

$$
K_{5}=\sum_{j=2}^{5} L_{1 j} \eta\left(z_{1}, z_{j}\right)+\sum_{2 \leq i<j \leq 5} K_{i j} \eta\left(z_{i}, z_{j}\right)
$$

where

$$
\begin{aligned}
L_{12} & =-\left\langle\left[\left(\lambda A^{1}\right)\left(k^{1} \cdot A^{2}\right)+A_{p}^{1}\left(\lambda \gamma^{p} W^{2}\right)\right]\left(\lambda \gamma^{m} W^{5}\right)\left(\lambda \gamma^{n} W^{3}\right) \mathcal{F}_{m n}^{4}\right\rangle \\
K_{25} & =-\left\langle\left(\lambda A^{1}\right)\left[\left(\lambda \gamma^{m} W^{2}\right)\left(k^{2} \cdot A^{5}\right)-\frac{1}{4}\left(\lambda \gamma^{m} \gamma^{r s} W^{5}\right) \mathcal{F}_{r s}^{2}\right]\left(\lambda \gamma^{n} W^{3}\right) \mathcal{F}_{m n}^{4}\right\rangle-(2 \leftrightarrow 5)
\end{aligned}
$$

and similarly for other choices of labels. The above two expressions can be understood as coming from the OPE computation of the natural generalization

$$
\left\langle\left(\lambda A^{1}\right)\left(\lambda \gamma^{m} W^{2}\right)\left(\lambda \gamma^{n} W^{3}\right) \mathcal{F}_{m n}^{4} U^{5}\right\rangle
$$

of the massless four-point kinematic factor $\left\langle\left(\lambda A^{1}\right)\left(\lambda \gamma^{m} W^{2}\right)\left(\lambda \gamma^{n} W^{3}\right) \mathcal{F}_{m n}^{4}\right\rangle$.

6 In the this paper we will simply refer to the non-minimal pure spinor formalism as "pure spinor formalism" (PS). 
In section 5 we calculate the (bosonic) component expansion of the "kinematic factor" (1.1) and show that it agrees, up to total derivative terms, with previous (bosonic 7 ) five-point results [28] 29] [30] [31]. We also compute some two- and four-fermion terms.

In Appendix A we review 6 the covariant (i.e. without relying on a U(5) decomposition as in [22]) proof of equivalence between the minimal and non-minimal one-loop massless four-point amplitude, as similar manipulations will be used again in the five-point kinematic factor computation of Appendix B. In Appendix C - for completeness - we show how to covariantly compute expressions of the form $\left\langle\lambda^{4} \bar{\lambda} \theta^{5}\right\rangle$ and in Appendix D we present the technicalities needed when computing component expansions of pure spinor superspace expressions. In particular, the kinematic reduction algorithms of [19] are expanded to deal with the structures appearing at five-points. They are well-suited for implementations using a computer algebra system such as FORM 33] 34 and/or Mathematica (using Ulf Gran's GAMMA package [35]).

\section{The scattering amplitude prescription}

The non-minimal pure spinor formalism prescription for the massless five-point open superstring amplitude at one-loop is given by [21]

$$
\mathcal{A}=\sum_{\text {top }} \int d t\left\langle\mathcal{N}(y)\left(\int d^{2} w \mu(w) b(w)\right) V^{1}(0) \prod_{I=2}^{5} \int d z_{I} U^{I}\left(z_{I}\right)\right\rangle
$$

Here $V^{1}(z)=\lambda^{\alpha} A_{\alpha}^{1}(x, \theta)$ denotes the unintegrated vertex operator and

$$
U(z)=\partial \theta^{\alpha} A_{\alpha}(x, \theta)+A_{m}(x, \theta) \Pi^{m}+d_{\alpha} W^{\alpha}(x, \theta)+\frac{1}{2} N_{m n} \mathcal{F}^{m n}(x, \theta)
$$

the integrated vertex operator, $\mu(w)$ is the Beltrami differential with conformal weight $(1,-1)$ and the sum is over the three Riemann surfaces which describe the one-loop interaction of open strings: the planar and non-planar cylinders and the Möbius strip. The b-ghost $b(w)$ is a composite field whose expression reads [21],

$$
b=s^{\alpha} \partial \bar{\lambda}_{\alpha}+\frac{2 \Pi^{m}\left(\bar{\lambda} \gamma_{m} d\right)-N_{m n}\left(\bar{\lambda} \gamma^{m n} \partial \theta\right)-J(\bar{\lambda} \partial \theta)-\left(\bar{\lambda} \partial^{2} \theta\right)}{4(\bar{\lambda} \lambda)}
$$

7 We also acknowledge the existence of fermionic results obtained by Lin et al. [26] using the methods of Atick and Sen [27], but we have not tried to bring their results to a form which could be readily compared to the fermionic results obtained from (1.1). As the pure spinor formalism is manifestly supersymmetric, we are guaranteed that once the bosonic amplitudes are verified to be correct then so are the fermionic ones.

8 This covariant proof was first obtained in 32. 


$$
+\frac{\left(\bar{\lambda} \gamma^{m n p} r\right)\left(d \gamma_{m n p} d+24 N_{m n} \Pi_{p}\right)}{192(\bar{\lambda} \lambda)^{2}}-\frac{\left(r \gamma^{m n p} r\right)\left(\bar{\lambda} \gamma_{m} d\right) N_{n p}}{16(\bar{\lambda} \lambda)^{3}}+\frac{\left(r \gamma^{m n p} r\right)\left(\bar{\lambda} \gamma_{p q r} r\right) N_{m n} N^{q r}}{128(\bar{\lambda} \lambda)^{4}} .
$$

The factor $\mathcal{N}(y)$ is needed to regularize the integration over the non-compact domain of the pure spinors, and we will use

$$
\mathcal{N}=\exp [-(\lambda \bar{\lambda})-(r \theta)-(w \bar{w})+(s d)]=\exp (\{Q, \chi\}) \text { for } \chi=-(\bar{\lambda} \theta)-(w s)
$$

This regularization is different from the one presented in [21] because our choice of $\chi$ is not gauge invariant. However, as it is still valid that $\mathcal{N}(y)=1+Q \Omega$ for some $\Omega$, the integral will be independent of the choice for $\chi$.

The evaluation of (2.1) will give rise to an expression of the form

$$
\mathcal{A}=\int[d \lambda][d \bar{\lambda}][d r][d w][d \bar{w}][d s]\left(d^{16} d\right) d^{16} \theta \mathcal{N} f(r, \theta),
$$

where the non-zero modes have already been integrated out through their OPEs. The zero modes are to be integrated with the following measures 9 :

$$
\begin{aligned}
{[d \lambda] } & =(\lambda \bar{\lambda})^{-3}\left(\epsilon_{16} \cdot d^{11} \lambda\right)_{\kappa_{1} \ldots \kappa_{5}}\left(\bar{\lambda} \gamma^{m}\right)^{\kappa_{1}}\left(\bar{\lambda} \gamma^{n}\right)^{\kappa_{2}}\left(\bar{\lambda} \gamma^{p}\right)^{\kappa_{3}}\left(\gamma_{m n p}\right)^{\kappa_{4} \kappa_{5}} \\
{[d \bar{\lambda}] } & =(\lambda \bar{\lambda})^{-3}\left(\epsilon_{16} \cdot d^{11} \bar{\lambda}\right)^{\kappa_{1} \ldots \kappa_{5}}\left(\lambda \gamma^{m}\right)_{\kappa_{1}}\left(\lambda \gamma^{n}\right)_{\kappa_{2}}\left(\lambda \gamma^{p}\right)_{\kappa_{3}}\left(\gamma_{m n p}\right)_{\kappa_{4} \kappa_{5}} \\
{[d r] } & =\epsilon_{\alpha_{1} \ldots \alpha_{11} \kappa_{1} \ldots \kappa_{5}}\left(\bar{\lambda} \gamma^{m}\right)^{\kappa_{1}}\left(\bar{\lambda} \gamma^{n}\right)^{\kappa_{2}}\left(\bar{\lambda} \gamma^{p}\right)^{\kappa_{3}} \gamma_{m n p}^{\kappa_{4} \kappa_{5}} \partial_{r}^{\alpha_{1}} \ldots \partial_{r}^{\alpha_{11}} \\
{[d w] } & =\left(\lambda \gamma^{m}\right)_{\kappa_{1}}\left(\lambda \gamma^{n}\right)_{\kappa_{2}}\left(\lambda \gamma^{p}\right)_{\kappa_{3}}\left(\gamma_{m n p}\right)_{\kappa_{4} \kappa_{5}} \epsilon^{\kappa_{1} \ldots \kappa_{5} \rho_{1} \ldots \rho_{11}} d w_{\rho_{1}} \ldots d w_{\rho_{11}} \\
{[d \bar{w}] } & =\left(\bar{\lambda} \gamma^{m}\right)^{\kappa_{1}}\left(\bar{\lambda} \gamma^{n}\right)^{\kappa_{2}}\left(\bar{\lambda} \gamma^{p}\right)^{\kappa_{3}}\left(\gamma_{m n p}\right)^{\kappa_{4} \kappa_{5}} \epsilon_{\kappa_{1} \ldots \kappa_{5} \alpha_{1} \ldots \alpha_{11}} d \bar{w}^{\alpha_{1}} \ldots d \bar{w}^{\alpha_{11}} \\
{[d s] } & =(\lambda \bar{\lambda})^{-3}\left(\lambda \gamma^{m}\right)_{\kappa_{1}}\left(\lambda \gamma^{n}\right)_{\kappa_{2}}\left(\lambda \gamma^{p}\right)_{\kappa_{3}}\left(\gamma_{m n p}\right)_{\kappa_{4} \kappa_{5}} \epsilon^{\kappa_{1} \ldots \kappa_{5} \rho_{1} \ldots \rho_{11}} \partial_{\rho_{1}}^{s} \ldots \partial_{\rho_{11}}^{s}
\end{aligned}
$$

Note that the measure (2.8) is gauge invariant under $\delta w_{\alpha}=\left(\lambda \gamma^{m}\right)_{\alpha} \Omega_{m}$ due to $\left(d \lambda \gamma^{q}\right)_{\left[\delta_{1}\right.}\left(\lambda \gamma^{m}\right)_{\kappa_{1}}\left(\lambda \gamma^{n}\right)_{\kappa_{2}}\left(\lambda \gamma^{p}\right)_{\kappa_{3}}\left(\gamma_{m n p}\right)_{\left.\kappa_{4} \kappa_{5}\right]}=0$, because there is no vector representation in the decomposition of $\lambda^{4} \theta^{6}$.

Apart from an overall coefficient which is not determined, one can use a shortcut to perform the integrations over $[d w],[d \bar{w}]$ and $[d s]$. At one loop there are eleven zero modes of $s^{\alpha}$, which can only 10 come from ten factors of the term $\left(s^{(0)} d^{(0)}\right)$ in the regulator $\mathcal{N}$.

\footnotetext{
9 They are written slightly differently from the original expressions of [21] (see e.g. 32])

10 The term $s^{\alpha} \partial \bar{\lambda}_{\alpha}$ of the b-ghost does not contribute because there is no $\bar{w}^{\alpha}$ in the external vertices to kill the non zero-modes of $\partial \bar{\lambda}_{\alpha}$.
} 
Therefore the remaining five $d_{\alpha}$ zero modes must come from the b-ghost and the external vertices. By ghost number conservation we obtain

$$
\int d^{16} d[d w][d \bar{w}][d s] \mathrm{e}^{-(w \bar{w})+(s d)-(\lambda \bar{\lambda})-(r \theta)} d_{\kappa_{1}}^{(0)} \ldots d_{\kappa_{5}}^{(0)} f^{\kappa_{1} \ldots \kappa_{5}}(r, \theta)=\left(\lambda^{3}\right)_{\left[\kappa_{1} \ldots \kappa_{5}\right]} f^{\kappa_{1} \ldots \kappa_{5}}(r, \theta)
$$

where $\left(\lambda^{3}\right)_{\left[\kappa_{1} \kappa_{2} \kappa_{3} \kappa_{4} \kappa_{5}\right]}$ is some tensor with five antisymmetric free indices containing three pure spinors. The unique such tensor is proportional to $\left(\lambda \gamma_{m}\right)_{\kappa_{1}}\left(\lambda \gamma_{n}\right)_{\kappa_{2}}\left(\lambda \gamma_{p}\right)_{\kappa_{3}}\left(\gamma^{m n p}\right)_{\kappa_{4} \kappa_{5}}$. One can thus see that the net effect of evaluating the pure spinor measures $[d w],[d \bar{w}],[d s]$ and $d^{16} d$ is to replace the five $d_{\alpha}^{(0)}$ zero-modes from the b-ghost and external vertices by

$$
d_{\kappa_{1}}^{(0)} d_{\kappa_{2}}^{(0)} d_{\kappa_{3}}^{(0)} d_{\kappa_{4}}^{(0)} d_{\kappa_{5}}^{(0)} \longrightarrow\left(\lambda \gamma_{m}\right)_{\kappa_{1}}\left(\lambda \gamma_{n}\right)_{\kappa_{2}}\left(\lambda \gamma_{p}\right)_{\kappa_{3}}\left(\gamma^{m n p}\right)_{\kappa_{4} \kappa_{5}}
$$

\section{The massless five-point amplitude computation}

\subsection{Zero mode saturation and OPEs}

The five $d_{\alpha}$ zero modes from the b-ghost and the external vertices appearing in (2.11) can be obtained by four distinct ways. Each one of them will imply a different "kinematic factor",

$$
\begin{aligned}
I_{1}= & \frac{1}{2}\left\langle\frac{\Pi^{m}\left(z_{0}\right)}{(\lambda \bar{\lambda})}\left(\bar{\lambda} \gamma_{m} d^{(0)}\right)\left(\lambda A^{1}\right)\left(d^{(0)} W^{2}\right)\left(d^{(0)} W^{3}\right)\left(d^{(0)} W^{4}\right)\left(d^{(0)} W^{5}\right)\right\rangle \\
I_{2}= & -\frac{1}{16}\left\langle\frac{\left(r \gamma_{m n p} r\right)}{(\lambda \bar{\lambda})^{3}}\left(\bar{\lambda} \gamma^{m} d^{(0)}\right) N^{n p}\left(z_{0}\right)\left(\lambda A^{1}\right)\left(d^{(0)} W^{2}\right)\left(d^{(0)} W^{3}\right)\left(d^{(0)} W^{4}\right)\left(d^{(0)} W^{5}\right)\right\rangle \\
I_{3}= & \frac{1}{96}\left\langle\frac{\left(\bar{\lambda} \gamma_{m n p} r\right)}{(\lambda \bar{\lambda})^{2}}\left(d^{(0)} \gamma^{m n p} \hat{d}\left(z_{0}\right)\right)\left(\lambda A^{1}\right)\left(d^{(0)} W^{2}\right)\left(d^{(0)} W^{3}\right)\left(d^{(0)} W^{4}\right)\left(d^{(0)} W^{5}\right)\right\rangle \\
I_{4}= & \frac{1}{192}\left\langle\frac{\left(\bar{\lambda} \gamma_{m n p} r\right)}{(\lambda \bar{\lambda})^{2}}\left(d^{(0)} \gamma^{m n p} d^{(0)}\right)\left(\lambda A^{1}\right)\left(d^{(0)} W^{2}\right)\left(d^{(0)} W^{3}\right)\left(d^{(0)} W^{4}\right)\right. \\
& \left.\times\left(A_{q}^{5} \Pi^{q}+\left(\hat{d} W^{5}\right)+\frac{1}{2} N^{m n} \mathcal{F}_{m n}^{5}\right)\right\rangle+\operatorname{cycl}(2345)
\end{aligned}
$$

where we have written $d_{\alpha}(z)=d_{\alpha}^{(0)} \omega(z)+\hat{d}_{\alpha}(z)$, with $\omega(z)$ being the holomorphic one-form and $d_{\alpha}^{(0)}$ the 16 zero modes of $d_{\alpha}$. In the expressions above one has to integrate out the conformal weight one variables through their OPEs and use the measures (2.5) - (2.10) to deal with the remaining zero modes, but we have omitted them as it is clear from the context that they are there 11 .

11 We also don't write the integration over the vertex positions in most of our formulae to avoid cluttering. One should note that the expressions we call "kinematic factors" also depend on the coordinates $z_{j}$ of the vertices, so that is a slight abuse of terminology. 
As discussed in [36], if the scalar propagator is $f(z, w)=\langle X(z, \bar{z}) X(w, \bar{w})\rangle$ then the OPE of a $(1,0)$-system with the zero modes projected out is given by $-\partial_{z} f(z, w)$. Furthermore one can show that up to terms which drop out of correlation functions [37] the function $f(z, w)$ in the torus is given in terms of the prime form $E(z, w)$ by

$$
f(z, w)=-\ln \left(|E(z, w)|^{2}\right)+2 \pi \frac{(\operatorname{Im}(z-w))^{2}}{\operatorname{Im} \tau},
$$

whereas for the cylinder and Möbius strip it is obtained through proper identifications under involutions [37]. We also define the $\eta(z, w)$ function by

$$
\langle\hat{d}(z) \theta(w)\rangle=-\langle\Pi(z) X(w, \bar{w})\rangle=-\frac{\partial}{\partial z} f(z, w) \equiv \eta(z, w),
$$

and the explicit expansions of both $E(z, w)$ and $\eta(z, w)$ in terms of Jacobi theta functions will not be needed here but they can be deduced from the formulae in [38].

\subsection{The vanishing of b-ghost OPEs}

We begin by noticing that after the OPEs are computed in $I_{1}, I_{2}$ and $I_{3}$ the result is actually a total derivative in $w$,

$$
\begin{aligned}
I_{1}+I_{2}+I_{3} & \propto \int d^{2} w \sum_{j=2}^{5} K_{0 j} \eta\left(w, z_{j}\right) \exp \left[\sum_{i<j}^{5}\left(k^{i} \cdot k^{j}\right) f\left(z_{i}, z_{j}\right)\right] \\
& \propto \int d^{2} w \frac{\partial}{\partial w}\left[\sum_{j=2}^{5} K_{0 j} f\left(w, z_{j}\right) \exp \left[\sum_{i<j}^{5}\left(k^{i} \cdot k^{j}\right) f\left(z_{i}, z_{j}\right)\right]\right],
\end{aligned}
$$

for some kinematic factors $K_{0 j}$, where $w$ and $z_{j}$ are the positions of the b-ghost and the external vertices, respectively. In (3.4) we used (3.3) and reinstated the integral over $w$ because it will be needed to prove that it vanishes for the topologies considered in (2.1). At this point it is instructive to see what one would obtain if the Riemann surface in (3.4) were the torus instead of, say, the cylinder. In this case the integration in (3.4) clearly vanishes because there can't be any contribution from non-trivial cycles, as the function $f\left(w, z_{j}\right)$ is doubly periodic over the torus. Now to see the vanishing of (3.4) for the cylinder one has to notice 12 that the b-ghost in $\int d^{2} w b(w)$ is already implicitly defined via the "doubling trick" to live in the double of the cylinder, which is the torus. So if the cylinder is the region [37]

$$
0 \leq \operatorname{Re}(w) \leq \pi, \quad w \sim w+2 \pi i t,
$$

12 CRM would like to thank Nathan Berkovits for discussions on this point. 
then both $b(w)$ and $\tilde{b}(\bar{w})$ are defined only in this region. The doubling trick in this case, however, consists in trading $b(w)$ and $\tilde{b}(\bar{w})$ by an augmented $b(w)$ defined also in the reflected region $-\pi \leq \operatorname{Re}(w) \leq 0$, through the following identification

$$
b(w)=\tilde{b}\left(\bar{w}^{\prime}\right), \quad-\pi \leq \operatorname{Re}(w) \leq 0, \quad w^{\prime}=-\bar{w} .
$$

Now the integral of (3.4) over this "doubled cylinder" results in two contributions over the boundaries at $\operatorname{Re}(w)= \pm \pi$, which cancel each other out because of the periodicity $f\left(w, z_{j}\right)=f\left(w+2 \pi, z_{j}\right)$.

\subsection{The kinematic factor in pure spinor superspace}

As shown above, there are no contributions from $I_{1}, \ldots, I_{3}$ and therefore the five-point kinematic factor is obtained entirely from $I_{4}$. This will be done by computing the OPEs between the conformal weight-one variables and using (2.11) to deal with the remaining five $d_{\alpha}$ zero-modes. Considering first the OPEs which do not involve the (fixed) position of the unintegrated vertex we restrict our attention to the terms proportional to e.g., $\eta\left(z_{2}, z_{5}\right)$,

$$
\begin{gathered}
\left\langle\frac{\left(\bar{\lambda} \gamma_{m n p} r\right)}{(\lambda \bar{\lambda})^{2}}\left(d \gamma^{m n p} d\right)\left(\lambda A^{1}\right)\left(d W^{2}\right)\left(d W^{3}\right)\left(d W^{4}\right)\left(A_{q}^{5} \Pi^{q}+\left(\hat{d} W^{5}\right)\right)\right\rangle+(2 \leftrightarrow 5) \\
=\eta\left(z_{5}, z_{2}\right) L_{52}+\eta\left(z_{2}, z_{5}\right) L_{25} \equiv \eta\left(z_{2}, z_{5}\right) K_{25}
\end{gathered}
$$

where, upon restoring the suppressed notation,

$$
\begin{gathered}
K_{25}=\int[d \lambda][d \bar{\lambda}][d r] \mathrm{e}^{-(\lambda \bar{\lambda})-(r \theta)} \frac{\left(\bar{\lambda} \gamma_{m n p} r\right)}{(\lambda \bar{\lambda})^{2}}\left[\frac{1}{4}\left(\lambda A^{1}\right)\left(\lambda \gamma^{m} \gamma^{r s} W^{5}\right)\left(\lambda \gamma^{n} W^{3}\right)\left(\lambda \gamma^{p} W^{4}\right) \mathcal{F}_{r s}^{2}\right. \\
\left.-k_{q}^{2}\left(\lambda A^{1}\right)\left(\lambda \gamma^{m} W^{2}\right)\left(\lambda \gamma^{n} W^{3}\right)\left(\lambda \gamma^{p} W^{4}\right) A_{q}^{5}\right]-(2 \leftrightarrow 5)
\end{gathered}
$$

To arrive at (3.5) one uses (2.11) and notes that $\eta\left(z_{2}, z_{5}\right)=-\eta\left(z_{5}, z_{2}\right)$. Finally from $r_{\alpha} \epsilon^{-(r \theta)}=-D_{\alpha} \epsilon^{-(r \theta)}$ one can replace $r_{\alpha}$ by $D_{\alpha}$ in (3.5). So we have expressed the oneloop kinematic factor computation in terms of a tree-level pure spinor superspace integral, which is amenable to various simplifications through the use of pure spinor identities. As will become clear during the computations, the presence of $\bar{\lambda}_{\alpha}$ in the superspace integral (3.5) will not play an important role: Using sufficiently many pure spinor superspace manipulations, they can always be seen to appear as factors of $(\lambda \bar{\lambda})$ in the terms which contribute to the kinematic factor 13

13 As a consequence - once we prove that the the $\bar{\lambda}_{\alpha} \lambda^{\beta}$ pair appear contracted as $(\lambda \bar{\lambda})$ - we essentially ignore their presence in our formulae, as they will only affect the overall coefficient. 
The task is now to compute

$$
\begin{aligned}
(\lambda \bar{\lambda})^{2} K_{25}= & \frac{1}{4}\left\langle\left(\bar{\lambda} \gamma_{m n p} D\right)\left[\left(\lambda A^{1}\right) \mathcal{F}_{r s}^{2}\left(\lambda \gamma^{m} \gamma^{r s} W^{5}\right)\left(\lambda \gamma^{n} W^{3}\right)\left(\lambda \gamma^{p} W^{4}\right)\right]\right\rangle \\
& -\left\langle\left(\bar{\lambda} \gamma_{m n p} D\right)\left[\left(k^{2} \cdot A^{5}\right)\left(\lambda A^{1}\right)\left(\lambda \gamma^{m} W^{2}\right)\left(\lambda \gamma^{n} W^{3}\right)\left(\lambda \gamma^{p} W^{4}\right)\right]\right\rangle-(2 \leftrightarrow 5) .
\end{aligned}
$$

As shown in Appendix B by using the action of the BRST operator

$$
Q \mathcal{F}_{m n}=2 k_{[m}\left(\lambda \gamma_{n]} W\right), \quad Q W^{\alpha}=\frac{1}{4}\left(\lambda \gamma^{m n}\right)^{\alpha} \mathcal{F}_{m n}, \quad Q A_{m}=\left(\lambda \gamma_{m} W\right)+k_{m}(\lambda A),
$$

the pure spinor constraint $\left(\lambda \gamma^{m} \lambda\right)=0$ and various gamma matrix identities we obtain

$$
\begin{aligned}
(\lambda \bar{\lambda})^{2} K_{25}= & -16\left\langle(\lambda \bar{\lambda})\left(\lambda A^{1}\right)\left(\lambda \gamma^{m} W^{2}\right)\left(\lambda \gamma^{n} W^{4}\right) \mathcal{F}_{m n}^{3}\left(k^{2} \cdot A^{5}\right)\right\rangle \\
& +4\left\langle(\lambda \bar{\lambda})\left(\lambda A^{1}\right)\left(\lambda \gamma^{m} \gamma^{r s} W^{5}\right)\left(\lambda \gamma^{n} W^{4}\right) \mathcal{F}_{r s}^{2} \mathcal{F}_{m n}^{3}\right\rangle \\
& -12\left\langle(\lambda \bar{\lambda})\left(\lambda A^{1}\right)\left(\lambda \gamma^{m} W^{2}\right)\left(\lambda \gamma^{n} W^{3}\right) \mathcal{F}_{m n}^{4}\left(k^{2} \cdot A^{5}\right)\right\rangle \\
& +3\left\langle(\lambda \bar{\lambda})\left(\lambda A^{1}\right)\left(\lambda \gamma^{m} \gamma^{r s} W^{5}\right)\left(\lambda \gamma^{n} W^{3}\right) \mathcal{F}_{r s}^{2} \mathcal{F}_{m n}^{4}\right\rangle \\
& -12\left\langle(\lambda \bar{\lambda})\left(\lambda A^{1}\right)\left(\lambda \gamma^{m} W^{3}\right)\left(\lambda \gamma^{n} W^{4}\right) \mathcal{F}_{m n}^{2}\left(k^{2} \cdot A^{5}\right)\right\rangle \\
& -24 k_{m}^{2}\left\langle(\lambda \bar{\lambda})\left(\lambda A^{1}\right)\left(\lambda \gamma^{[m} W^{3}\right)\left(\lambda \gamma^{n]} W^{4}\right)\left(W^{5} \gamma_{n} W^{2}\right)\right\rangle \\
& -12\left\langle(\lambda \bar{\lambda})\left(\lambda A^{1}\right)\left(\lambda \gamma^{[m} W^{3}\right)\left(\lambda \gamma^{n]} W^{4}\right) \mathcal{F}_{m t}^{2} \mathcal{F}_{n t}^{5}\right\rangle \\
& +2\left(k^{2} \cdot k^{5}\right)\left\langle\left(\lambda A^{1}\right)\left(\lambda A^{5}\right)\left(\lambda \gamma^{m} W^{2}\right)\left(\lambda \gamma^{n} W^{4}\right)\left(\bar{\lambda} \gamma_{m n} W^{3}\right)\right\rangle \\
& +2\left(k^{2} \cdot k^{5}\right)\left\langle(\lambda \bar{\lambda})\left(\lambda A^{5}\right)\left(\lambda \gamma^{m} W^{2}\right)\left(\lambda \gamma^{n} W^{4}\right)\left(A^{1} \gamma_{m n} W^{3}\right)\right\rangle \\
& -2\left(k^{2} \cdot k^{5}\right)\left\langle(\lambda \bar{\lambda})\left(\lambda A^{1}\right)\left(\lambda \gamma^{m} W^{2}\right)\left(\lambda \gamma^{n} W^{4}\right)\left(A^{5} \gamma_{m n} W^{3}\right)\right\rangle-(2 \leftrightarrow 5)
\end{aligned}
$$

As mentioned before, the pure spinor $\bar{\lambda}_{\alpha}$ appears only in overall factors of $(\lambda \bar{\lambda})$ except for one term - which will turn out to be part of a total derivative, as also shown in Appendix B. However (3.7) is not a "simple" expression by any standard, and it would be desirable to seek for cancellations of terms by manipulations in pure spinor superspace. This will be done after we prove the gauge invariance of the amplitude.

\subsection{Gauge invariance}

The one-loop scattering amplitude prescription in the non-minimal pure spinor formalism [21] uses one non-integrated vertex operator and therefore is not manifestly gauge invariant. Nevertheless gauge invariance can be proved after the integration over the vertex operator positions is performed, as one can easily verify from the prescription. One 
can also see this explicitly for the kinematic factor (3.7) as follows. From the five-point amplitude prescription of (2.1) it follows that under the gauge variation of $\delta A_{\alpha}^{1}=D_{\alpha} \Omega^{1}$,

$$
\delta \mathcal{A}=\int d t\left\langle\mathcal{N} \int(b \cdot \mu) \Omega^{1} \int U^{2} \int U^{3} \int U^{4} \int \partial\left(\lambda A^{5}\right)\right\rangle+\operatorname{cycl}(2345)=0 .
$$

In the above we "integrated the BRST charge by parts", used the identity $Q U^{5}=\partial\left(\lambda A^{5}\right)$ [8] and the fact that the contribution from $\{Q, b\}=T$ vanishes identically due to the lack of $d_{\alpha}$ zero modes [22]. Furthermore, the vanishing of (3.8) is justified by the cancelled propagator argument. Using $\partial\left(\lambda A^{5}\right)=\partial \theta^{\alpha} \partial_{\alpha}\left(\lambda A^{5}\right)+\Pi^{m} k_{m}^{5}\left(\lambda A^{5}\right)$ one gets the terms proportional to $\eta\left(z_{2}, z_{5}\right)$

$$
\left(k^{2} \cdot k^{5}\right)\left\langle\left(\bar{\lambda} \gamma_{m n p} D\right)\left[\Omega^{1}\left(\lambda A^{5}\right)\left(\lambda \gamma^{m} W^{2}\right)\left(\lambda \gamma^{n} W^{3}\right)\left(\lambda \gamma^{p} W^{4}\right)\right]\right\rangle-(2 \leftrightarrow 5) \simeq 0
$$

where we used (2.11). The $\simeq$ sign is to remember that the expression vanishes after integration over the positions (we omitted the integral signs to avoid cluttering). Similar manipulations to the ones of appendix A lead to

$$
\begin{aligned}
& \left\langle\left(\bar{\lambda} \gamma_{m n p} D\right)\left[\Omega^{1}\left(\lambda A^{5}\right)\left(\lambda \gamma^{m} W^{2}\right)\left(\lambda \gamma^{n} W^{3}\right)\left(\lambda \gamma^{p} W^{4}\right)\right]\right\rangle \\
= & \left\langle\left(\bar{\lambda} \gamma_{m n p} D\right)\left[\left(\lambda A^{5}\right) \Omega^{1}\right]\left(\lambda \gamma^{m} W^{2}\right)\left(\lambda \gamma^{n} W^{3}\right)\left(\lambda \gamma^{p} W^{4}\right)\right\rangle \\
+ & 12(\lambda \bar{\lambda})\left\langle\Omega^{1}\left(\lambda A^{5}\right)\left[\left(\lambda \gamma^{m} W^{2}\right)\left(\lambda \gamma^{n} W^{3}\right) \mathcal{F}_{m n}^{4}+\operatorname{cycl}(234)\right]\right\rangle
\end{aligned}
$$

and

$$
\begin{aligned}
& \left\langle\left(\bar{\lambda} \gamma_{m n p} D\right)\left[\left(\lambda A^{5}\right) \Omega^{1}\right]\left(\lambda \gamma^{m} W^{2}\right)\left(\lambda \gamma^{n} W^{3}\right)\left(\lambda \gamma^{p} W^{4}\right)\right\rangle \\
= & 4(\lambda \bar{\lambda})\left\langle\Omega^{1}\left(\lambda A^{5}\right)\left(\lambda \gamma^{m} W^{2}\right)\left(\lambda \gamma^{n} W^{4}\right) \mathcal{F}_{m n}^{3}\right\rangle \\
+ & 2\left\langle\left(Q \Omega^{1}\right)\left(\lambda \gamma^{m} W^{2}\right)\left(\lambda \gamma^{n} W^{4}\right)\left[\left(\lambda A^{5}\right)\left(\bar{\lambda} \gamma_{m n} W^{3}\right)-(\lambda \bar{\lambda})\left(A^{5} \gamma_{m n} W^{3}\right)\right]\right\rangle \\
+ & 2\left\langle(\lambda \bar{\lambda})\left(\lambda A^{5}\right)\left(\lambda \gamma^{m} W^{2}\right)\left(\lambda \gamma^{n} W^{4}\right)\left(W^{3} \gamma_{m n} D\right) \Omega^{1}\right\rangle .
\end{aligned}
$$

From $(3.9),(3.10)$ and $(\underline{3.11})$ it follows that $\delta \mathcal{A}=\eta\left(z_{2}, z_{5}\right) \delta K_{25} \simeq 0$, where

$$
\begin{aligned}
(\lambda \bar{\lambda}) \delta K_{25}= & +16\left(k^{2} \cdot k^{5}\right)\left\langle\Omega^{1}\left(\lambda A^{5}\right)\left(\lambda \gamma^{m} W^{2}\right)\left(\lambda \gamma^{n} W^{4}\right) \mathcal{F}_{m n}^{3}\right\rangle \\
& +12\left(k^{2} \cdot k^{5}\right)\left\langle\Omega^{1}\left(\lambda A^{5}\right)\left(\lambda \gamma^{m} W^{2}\right)\left(\lambda \gamma^{n} W^{3}\right) \mathcal{F}_{m n}^{4}\right\rangle \\
& +12\left(k^{2} \cdot k^{5}\right)\left\langle\Omega^{1}\left(\lambda A^{5}\right)\left(\lambda \gamma^{m} W^{3}\right)\left(\lambda \gamma^{n} W^{4}\right) \mathcal{F}_{m n}^{2}\right\rangle \\
& +2\left(k^{2} \cdot k^{5}\right)\left\langle\left(Q \Omega^{1}\right)\left(\lambda A^{5}\right)\left(\lambda \gamma^{m} W^{2}\right)\left(\lambda \gamma^{n} W^{4}\right)\left(\bar{\lambda} \gamma_{m n} W^{3}\right)\right\rangle \\
& +2\left(k^{2} \cdot k^{5}\right)\left\langle\left(\lambda A^{5}\right)\left(\lambda \gamma^{m} W^{2}\right)\left(\lambda \gamma^{n} W^{4}\right)\left(W^{3} \gamma_{m n} D\right) \Omega^{1}\right\rangle \\
& -2\left(k^{2} \cdot k^{5}\right)\left\langle\left(Q \Omega^{1}\right)\left(\lambda \gamma^{m} W^{2}\right)\left(\lambda \gamma^{n} W^{4}\right)\left(A^{5} \gamma_{m n} W^{3}\right)\right\rangle-(2 \leftrightarrow 5) .
\end{aligned}
$$


Now it remains to be shown that (3.12) is identical to the gauge variation of (3.7). To see this one uses

$$
\begin{gathered}
\left\langle\left(Q \Omega^{1}\right)\left(\lambda \gamma^{m} W^{2}\right)\left(\lambda \gamma^{n} W^{4}\right) \mathcal{F}_{m n}^{3}\left(k^{2} \cdot A^{5}\right)\right\rangle \\
=-\left\langle\Omega^{1}\left(\lambda \gamma^{m} W^{2}\right)\left(\lambda \gamma^{n} W^{4}\right) \mathcal{F}_{m n}^{3}\left[k_{r}^{2}\left(\lambda \gamma^{r} W^{5}\right)+\left(k^{2} \cdot k^{5}\right)\left(\lambda A^{5}\right)\right]\right\rangle,
\end{gathered}
$$

and

$$
\begin{gathered}
\left\langle\left(Q \Omega^{1}\right)\left(\lambda \gamma^{m} \gamma^{r s} W^{5}\right)\left(\lambda \gamma^{n} W^{4}\right) \mathcal{F}_{r s}^{2} \mathcal{F}_{m n}^{3}\right\rangle-(2 \leftrightarrow 5) \\
=-4 k_{r}^{2}\left\langle\Omega^{1}\left(\lambda \gamma^{r} W^{5}\right)\left(\lambda \gamma^{m} W^{4}\right)\left(\lambda \gamma^{n} W^{2}\right) \mathcal{F}_{m n}^{3}\right\rangle-(2 \leftrightarrow 5),
\end{gathered}
$$

where the explicit antisymmetry in [25] is important, otherwise there would be extra terms. Furthermore,

$$
\begin{gathered}
-k_{m}^{2}\left\langle\left(Q \Omega^{1}\right)\left(\lambda \gamma^{[m} W^{3}\right)\left(\lambda \gamma^{n]} W^{4}\right)\left(W^{5} \gamma_{n} W^{2}\right)\right\rangle \\
=-k_{m}^{2}\left\langle\Omega^{1}\left(\lambda \gamma^{[m} W^{3}\right)\left(\lambda \gamma^{n]} W^{4}\right)\left[\left(\lambda \gamma^{t} W^{2}\right) \mathcal{F}_{n t}^{5}-\left(\lambda \gamma^{t} W^{5}\right) \mathcal{F}_{n t}^{2}\right]\right\rangle
\end{gathered}
$$

and finally

$$
\begin{gathered}
-\left\langle\left(Q \Omega^{1}\right)\left(\lambda \gamma^{[m} W^{3}\right)\left(\lambda \gamma^{n]} W^{4}\right) \mathcal{F}_{m t}^{2} \mathcal{F}_{n t}^{5}\right\rangle \\
=+\left\langle\Omega^{1}\left(\lambda \gamma^{[m} W^{3}\right)\left(\lambda \gamma^{n]} W^{4}\right)\left[\left(\lambda \gamma^{t} W^{2}\right) k_{m}^{2} \mathcal{F}_{n t}^{5}-\left(\lambda \gamma^{t} W^{5}\right) k_{m}^{5} \mathcal{F}_{n t}^{2}\right]\right\rangle
\end{gathered}
$$

From the above results one obtains the gauge variation of (3.7). It reads

$$
\begin{aligned}
(\lambda \bar{\lambda}) \delta K_{25}= & +16\left(k^{2} \cdot k^{5}\right)\left\langle\Omega^{1}\left(\lambda A^{5}\right)\left(\lambda \gamma^{m} W^{2}\right)\left(\lambda \gamma^{n} W^{4}\right) \mathcal{F}_{m n}^{3}\right\rangle \\
& +12\left(k^{2} \cdot k^{5}\right)\left\langle\Omega^{1}\left(\lambda A^{5}\right)\left(\lambda \gamma^{m} W^{2}\right)\left(\lambda \gamma^{n} W^{3}\right) \mathcal{F}_{m n}^{4}\right\rangle \\
& +12\left(k^{2} \cdot k^{5}\right)\left\langle\Omega^{1}\left(\lambda A^{5}\right)\left(\lambda \gamma^{m} W^{3}\right)\left(\lambda \gamma^{n} W^{4}\right) \mathcal{F}_{m n}^{2}\right\rangle \\
& +2\left(k^{2} \cdot k^{5}\right)\left\langle\left(Q \Omega^{1}\right)\left(\lambda A^{5}\right)\left(\lambda \gamma^{m} W^{2}\right)\left(\lambda \gamma^{n} W^{4}\right)\left(\bar{\lambda} \gamma_{m n} W^{3}\right)\right\rangle \\
& +2\left(k^{2} \cdot k^{5}\right)\left\langle\left(\lambda A^{5}\right)\left(\lambda \gamma^{m} W^{2}\right)\left(\lambda \gamma^{n} W^{4}\right)\left(W^{3} \gamma_{m n} D\right) \Omega^{1}\right\rangle \\
& -2\left(k^{2} \cdot k^{5}\right)\left\langle\left(Q \Omega^{1}\right)\left(\lambda \gamma^{m} W^{2}\right)\left(\lambda \gamma^{n} W^{4}\right)\left(A^{5} \gamma_{m n} W^{3}\right)\right\rangle-(2 \leftrightarrow 5)
\end{aligned}
$$

and it is equal to (3.12), as was expected. To get (3.16) from (3.7) we also used that $k_{r}^{2}\left\langle\Omega^{1}\left(\lambda \gamma^{r} W^{5}\right)\left(\lambda \gamma^{m} W^{3}\right)\left(\lambda \gamma^{n} W^{4}\right) \mathcal{F}_{m n}^{2}\right\rangle+2 k_{m}^{2}\left\langle\Omega^{1}\left(\lambda \gamma^{[m} W^{3}\right)\left(\lambda \gamma^{n]} W^{4}\right)\left(\lambda \gamma^{t} W^{5}\right) \mathcal{F}_{n t}^{2}\right\rangle=0$,

which can be proved by writing $\mathcal{F}_{n t}^{2}=k_{n}^{2} A_{t}^{2}-k_{t}^{2} A_{n}^{2}$ and noting the vanishing of the factor containing $k_{m}^{2} k_{n}^{2}$ due to the antisymmetry over $[m n]$. Therefore the second term of (3.17) is equal to

$$
-2\left\langle\Omega^{1}\left(\lambda \gamma^{[m} W^{3}\right)\left(\lambda \gamma^{n]} W^{4}\right)\left(\lambda \gamma^{t} W^{5}\right) k_{m}^{2} k_{t}^{2} A_{n}^{2}\right\rangle=-k_{t}^{2}\left\langle\Omega^{1}\left(\lambda \gamma^{t} W^{5}\right)\left(\lambda \gamma^{m} W^{3}\right)\left(\lambda \gamma^{n} W^{4}\right) \mathcal{F}_{m n}^{2}\right\rangle,
$$

which cancels the first. 


\section{Simplifying the answer}

As one can see from (3.7) the kinematic factor is a bit awkward and it would be desirable to simplify it using pure spinor superspace identities. This is accomplished in Appendix B, where among other things we identified and dropped total derivative terms in (3.7), which simplifies to

$$
K_{25}=-40\left\langle\left(\lambda A^{1}\right)\left[\left(\lambda \gamma^{m} W^{2}\right)\left(k^{2} \cdot A^{5}\right)-\frac{1}{4}\left(\lambda \gamma^{m} \gamma^{r s} W^{5}\right) \mathcal{F}_{r s}^{2}\right]\left(\lambda \gamma^{n} W^{3}\right) \mathcal{F}_{m n}^{4}\right\rangle-(2 \leftrightarrow 5) .
$$

One should note the absence of $\bar{\lambda}_{\alpha}$ in the rhs of (4.1), which is a remarkable fact in view of (3.5). That makes of (4.1) a truly tree-level pure spinor superspace integral, which is readily evaluated using the measure $\left\langle\left(\lambda \gamma^{m} \theta\right)\left(\lambda \gamma^{n} \theta\right)\left(\lambda \gamma^{p} \theta\right)\left(\theta \gamma_{m n p} \theta\right)\right\rangle=1$ and the method described in the appendix of [22] (see also other methods in [19]). The gauge transformation of (4.1) under $\delta A_{\alpha}^{1}=D_{\alpha} \Omega^{1}$ is simply

$$
(\lambda \bar{\lambda}) \delta K_{25}=40\left(k^{2} \cdot k^{5}\right)\left\langle\Omega^{1}\left(\lambda A^{5}\right)\left(\lambda \gamma^{m} W^{2}\right)\left(\lambda \gamma^{n} W^{3}\right) \mathcal{F}_{m n}^{4}\right\rangle-(2 \leftrightarrow 5),
$$

where we used $\left(\lambda \gamma^{m} \gamma^{r s} W^{5}\right) k_{r}^{2}\left(\lambda \gamma_{s} W^{2}\right)=2 k_{r}^{2}\left(\lambda \gamma^{m} W^{2}\right)\left(\lambda \gamma^{r} W^{5}\right)$ [20]. Furthermore one can observe that (4.1) is the result of computing the $z_{5} \rightarrow z_{2}$ OPE in

$$
40\left\langle\left(\lambda A^{1}\right)\left(\lambda^{0} \gamma^{m} W^{2}\right)\left(\lambda^{0} \gamma^{n} W^{3}\right) \mathcal{F}_{m n}^{4} U^{5}\right\rangle
$$

with the restriction that the pure spinors in $\left(\lambda \gamma^{m} W^{2}\right)$ and $\left(\lambda \gamma^{n} W^{3}\right)$ have no non-zero modes, but $\left(\lambda A^{1}\right)$ has. These $\lambda^{\alpha}$ non zero-modes can be understood by noticing that except for the pure spinor appearing in the non-integrated vertex - which comes from the amplitude prescription - the others are the zero-modes of $\lambda^{\alpha}$ which appear in the measures (2.5) - (2.10). Having found (4.3), the generalization to the scattering of closed strings almost suggests itself and could be used to check the $\left(t_{8} t_{8} \pm \epsilon_{10} \epsilon_{10}\right) R^{4}$ sign issue (see e.g. [29]).

Motivated by the simplicity of (4.3), we now investigate whether this single correlator can reproduce the other contributions to the amplitude as well. Using (4.3) and the identities of [20] one can easily compute the OPEs involving the position of the unintegrated vertex. For example, the computation as $z_{5} \rightarrow z_{1}$ leads to

$$
L_{15}=-40\left\langle\left[\left(\lambda A^{1}\right)\left(k^{1} \cdot A^{5}\right)+A_{p}^{1}\left(\lambda \gamma^{p} W^{5}\right)\right]\left(\lambda \gamma^{m} W^{2}\right)\left(\lambda \gamma^{n} W^{3}\right) \mathcal{F}_{m n}^{4}\right\rangle
$$


whose gauge transformation under $\delta A_{\alpha}^{1}=D_{\alpha} \Omega^{1}$ can be easily checked to be

$$
\delta L_{15}=+40\left(k^{1} \cdot k^{5}\right)\left\langle\Omega^{1}\left(\lambda A^{5}\right)\left(\lambda \gamma^{m} W^{2}\right)\left(\lambda \gamma^{n} W^{3}\right) \mathcal{F}_{m n}^{4}\right\rangle
$$

With these explicit results for the OPEs involving the position $z_{1}$ we can obtain an expression for the full kinematic factor whose gauge invariance is easily checked. To do this we use that

$$
L_{15} \eta(1,5)=-L_{15} \frac{1}{\left(k^{1} \cdot k^{5}\right)}\left[\left(k^{2} \cdot k^{5}\right) \eta(2,5)+\left(k^{3} \cdot k^{5}\right) \eta(3,5)+\left(k^{4} \cdot k^{5}\right) \eta(4,5)\right]
$$

(similarly for $\left.L_{1 j}\right)$ which follows from the vanishing of $\int L_{15}\left(\partial / \partial z_{5}\right) \exp \sum\left(k^{i} \cdot k^{j}\right) f\left(z_{i}, z_{j}\right)$ using the canceled propagator argument. This allows one to express terms proportional to $\eta\left(z_{1}, z_{j}\right)$ as a linear combination of $\eta\left(z_{i}, z_{j}\right)$, where $i, j \neq 1$. Doing this one obtains

$$
K_{5}=\sum_{2 \leq i<j \leq 5}\left[K_{i j}-L_{1 j} \frac{\left(k^{i} \cdot k^{j}\right)}{\left(k^{1} \cdot k^{j}\right)}+L_{1 i} \frac{\left(k^{i} \cdot k^{j}\right)}{\left(k^{1} \cdot k^{i}\right)}\right] \eta\left(z_{i}, z_{j}\right)
$$

whose gauge invariance is easily shown by using (4.2), (4.5) and the analogous expressions for the other factors of $K_{i j}$ and $L_{1 j}$. For completeness we also write down the result for the $L_{15}$ kinematic factor which follows from the analogous 14 and equally long computation leading to 3.7 ,

$$
\begin{aligned}
(\lambda \bar{\lambda})^{2} L_{15}= & -16(\lambda \bar{\lambda})\left\langle\left(\lambda A^{1}\right)\left(\lambda \gamma^{m} W^{2}\right)\left(\lambda \gamma^{n} W^{4}\right) \mathcal{F}_{m n}^{3}\left(k^{1} \cdot A^{5}\right)\right\rangle \\
& -12(\lambda \bar{\lambda})\left\langle\left(\lambda A^{1}\right)\left(\lambda \gamma^{m} W^{2}\right)\left(\lambda \gamma^{n} W^{3}\right) \mathcal{F}_{m n}^{4}\left(k^{1} \cdot A^{5}\right)\right\rangle \\
& -12(\lambda \bar{\lambda})\left\langle\left(\lambda A^{1}\right)\left(\lambda \gamma^{m} W^{3}\right)\left(\lambda \gamma^{n} W^{4}\right) \mathcal{F}_{m n}^{2}\left(k^{1} \cdot A^{5}\right)\right\rangle \\
& -16(\lambda \bar{\lambda})\left\langle A_{p}^{1}\left(\lambda \gamma^{m} W^{2}\right)\left(\lambda \gamma^{n} W^{4}\right) \mathcal{F}_{m n}^{3}\left(\lambda \gamma^{p} W^{5}\right)\right\rangle \\
& -12(\lambda \bar{\lambda})\left\langle A_{p}^{1}\left(\lambda \gamma^{m} W^{2}\right)\left(\lambda \gamma^{n} W^{3}\right) \mathcal{F}_{m n}^{4}\left(\lambda \gamma^{p} W^{5}\right)\right\rangle \\
& -12(\lambda \bar{\lambda})\left\langle A_{p}^{1}\left(\lambda \gamma^{m} W^{3}\right)\left(\lambda \gamma^{n} W^{4}\right) \mathcal{F}_{m n}^{2}\left(\lambda \gamma^{p} W^{5}\right)\right\rangle \\
& +2\left(k^{1} \cdot k^{5}\right)\left\langle\left(\lambda A^{1}\right)\left(\lambda A^{5}\right)\left(\lambda \gamma^{m} W^{2}\right)\left(\lambda \gamma^{n} W^{4}\right)\left(\bar{\lambda} \gamma_{m n} W^{3}\right)\right\rangle \\
& +2(\lambda \bar{\lambda})\left(k^{1} \cdot k^{5}\right)\left\langle\left(\lambda A^{5}\right)\left(\lambda \gamma^{m} W^{2}\right)\left(\lambda \gamma^{n} W^{4}\right)\left(A^{1} \gamma_{m n} W^{3}\right)\right\rangle \\
& -2(\lambda \bar{\lambda})\left(k^{2} \cdot k^{5}\right)\left\langle\left(\lambda A^{1}\right)\left(\lambda \gamma^{m} W^{2}\right)\left(\lambda \gamma^{n} W^{4}\right)\left(A^{5} \gamma_{m n} W^{3}\right)\right\rangle .
\end{aligned}
$$

14 The actual details are different because it involves a different set of OPEs to be computed, which also includes the contribution from $\frac{1}{2} N^{m n} \mathcal{F}_{m n}$. 
We have checked that the component expansion of (4.6) does not change if we use the simplified expressions (4.1) and (4.4) or the longer versions given by (3.7) and (4.7) (and their analogous expressions for other labels). That provides a consistency check that the total derivative terms were correctly identified.

We conclude that the complete five-point amplitude is given by

$$
\mathcal{A}=\sum_{\text {top }} \int \frac{d t}{t} \prod_{I=2}^{5} \int d z^{I} K_{5}\left(z_{1}, \ldots, z_{5}\right)\left\langle\prod_{i=1}^{5} \mathrm{e}^{i k^{i} \cdot X\left(z_{i}, \bar{z}_{i}\right)}\right\rangle
$$

where - as alluded to in the introduction - the kinematic factor is

$$
K_{5}\left(z_{1}, \ldots, z_{5}\right)=\sum_{j=2}^{5} L_{1 j} \eta\left(z_{1}, z_{j}\right)+\sum_{2 \leq i<j \leq 5} K_{i j} \eta\left(z_{i}, z_{j}\right)
$$

and has the explicit form

$$
\begin{aligned}
& L_{12}=-40\left\langle\left[\left(\lambda A^{1}\right)\left(k^{1} \cdot A^{2}\right)+A_{p}^{1}\left(\lambda \gamma^{p} W^{2}\right)\right]\left(\lambda \gamma^{m} W^{5}\right)\left(\lambda \gamma^{n} W^{3}\right) \mathcal{F}_{m n}^{4}\right\rangle \\
& K_{25}=-40\left\langle\left(\lambda A^{1}\right)\left[\left(\lambda \gamma^{m} W^{2}\right)\left(k^{2} \cdot A^{5}\right)-\frac{1}{4}\left(\lambda \gamma^{m} \gamma^{r s} W^{5}\right) \mathcal{F}_{r s}^{2}\right]\left(\lambda \gamma^{n} W^{3}\right) \mathcal{F}_{m n}^{4}\right\rangle-(2 \leftrightarrow 5)
\end{aligned}
$$

and analogously for the other labels. Furthermore, as mentioned above, these kinematic expressions can be obtained from (4.3), which encapsulates all the information about the kinematic structures appearing at five-points.

\section{Bosonic and fermionic component expansions}

\subsection{Matching with RNS, GS and Lee \& Siegel}

The superspace results of the previous section summarize the computation using the pure spinor formalism. The simplicity of (4.9) is strong evidence of its correctness, but it must nevertheless be compared with previous results [26] 29] 30] 31] obtained with the RNS and GS formalisms and the "ghost pyramid" approach by Lee and Siegel (LS) [28].

The most straightforward comparison turned out to be with the five-graviton amplitude calculation by Richards [29], thanks to the clarity of the results presented there. The open-string parts of that closed-string calculation are clearly identified and take the form of a kinematic factor $A_{r s}$ (see eq. (3.20) of [29]). Even though the tensors $A_{i j}$ from Richards and our expressions for $K_{i j}$ (and similarly $L_{1 j}$ ) are not exactly the same (they differ by $\left(k^{i} \cdot k^{j}\right)$ terms) one can check that the gauge invariant expression (4.6) does not 
change when $K_{i j}$ is substituted by $A_{i j}$. The conclusion is that the difference is due to total derivative terms, as they are automatically eliminated in (4.6). The pure spinor formalism is thus equivalent to the (bosonic) GS result of [29], and being a supersymmetric formalism we are guaranteed that the scattering amplitudes involving fermionic external states are also the correct ones.

The comparison with the (bosonic) kinematic factor computed by Lee and Siegel [28] is also done by discarding total derivative terms. One can easily see the need for that by noticing that their result features a manifestly gauge invariant integrand (see e.g. their equation (5.5.1)), whereas ours is gauge invariant only after integration over the vertex positions. Therefore one should expect a matching only after total derivative terms are properly taken into account. Doing that carefully, we obtain agreement with LS.

More precisely, we first checked that the pole terms of our expressions agree exactly with the pole terms of equations (5.5.1) and (5.5.2) in [28]. This is expected because they are not affected by total derivative ambiguities. We compared the non-pole terms which are proportional to $\eta\left(z_{2}, z_{5}\right)$, and they are completely accounted for by adding a total derivative term in superspace. To see this explicitly one can add a specially crafted total derivative term to (4.1) as follows:

$$
\begin{gathered}
(\lambda \bar{\lambda}) K_{25}=L_{25}-L_{52} \\
=-40\left\langle\left(\lambda A^{1}\right)\left(\lambda \gamma^{m} W^{2}\right)\left(\lambda \gamma^{n} W^{4}\right) \mathcal{F}_{m n}^{3}\left(k^{2} \cdot A^{5}\right)\right\rangle+10\left\langle\left(\lambda A^{1}\right)\left(\lambda \gamma^{m} \gamma^{r s} W^{5}\right)\left(\lambda \gamma^{n} W^{4}\right) \mathcal{F}_{r s}^{2} \mathcal{F}_{m n}^{3}\right\rangle \\
+20\left(k^{2} \cdot k^{5}\right)\left\langle\left(\lambda A^{5}\right)\left(\lambda \gamma^{m} W^{2}\right)\left(\lambda \gamma^{n} W^{4}\right)\left(A^{1} \gamma_{m n} W^{3}\right)\right\rangle-(2 \leftrightarrow 5) .
\end{gathered}
$$

The last line is a total derivative in $z_{5}$ : it comes from

$$
+20\left\langle\left(\lambda \gamma^{m} W^{2}\right)\left(\lambda \gamma^{n} W^{4}\right)\left(A^{1} \gamma_{m n} W^{3}\right) \partial\left(\lambda A^{5}\right)\right\rangle
$$

as can be seen using $\partial\left(\lambda A^{5}\right)=\partial \theta^{\alpha} \partial_{\alpha}\left(\lambda A^{5}\right)+\Pi^{m} k_{m}^{5}\left(\lambda A^{5}\right)$. By explicit computation one can then show that the five gluon component expansion of (5.1) is given by the simple expression

$$
\begin{aligned}
K_{25}^{5 b}= & +\frac{1}{576} t_{8}^{m n p q r s t u} F_{m n}^{1} F_{p a}^{2} F_{r s}^{3} F_{t u}^{4} F_{q a}^{5} \\
& +\frac{1}{1152} t_{8}^{m n p q r s t u}\left[\left(k^{2} \cdot e^{5}\right) F_{m n}^{1} F_{p q}^{2} F_{r s}^{3} F_{t u}^{4}-\left(k^{5} \cdot e^{2}\right) F_{m n}^{1} F_{p q}^{5} F_{r s}^{3} F_{t u}^{4}\right]
\end{aligned}
$$

which is the same answer one gets from evaluating the traces in [28]. We therefore conclude the equivalence of the PS and LS results. 
Adopting the "Schoonschip" notation by writing objects contracted into $t_{8}$ directly on the tensor rather than using dummy indices, we now rewrite (5.2) in terms of polarizations,

$$
\begin{aligned}
& K_{25}^{5 b}=\frac{1}{72}\left[\left(k^{2} \cdot e^{5}\right) t_{8}^{e^{2}\left(k^{2}+k^{5}\right) e^{1} k^{1} e^{3} k^{3} e^{4} k^{4}}-\left(k^{5} \cdot e^{2}\right) t_{8}^{e^{5}\left(k^{2}+k^{5}\right) e^{1} k^{1} e^{3} k^{3} e^{4} k^{4}}\right.
\end{aligned}
$$

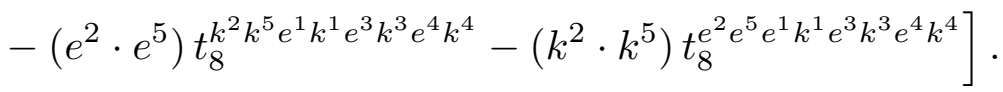

This is, up to an overall coefficient, the same expression as Richards' $A_{25}$ - therefore also confirming the equivalence between the LS and the light-cone GS calculations. It is reassuring to see that everything matches.

Furthermore one can exploit the superfield nature of the pure spinor result (5.1) to obtain the component expansion involving fermionic fields. For example, the $b_{1} f_{2} b_{3} b_{4} f_{5}$ expansion of (5.1) is given by

$$
K_{25}^{2 f 3 b}=\frac{1}{576} t_{8}^{m n p q r s t u}\left(k_{m}^{2}+k_{m}^{5}\right)\left(\chi^{2} \gamma_{n} \chi^{5}\right) F_{p q}^{1} F_{r s}^{3} F_{t u}^{4} .
$$

All the other fermionic component expansions are also easily obtained. However we should note that although (5.2) and (5.4) happen to be gauge invariant, that is not true for other combination of external particles, and one really needs to use (4.6) to get explicitly gauge invariant results using (5.1).

\subsection{A different superspace expression for the kinematic factor $K_{i j}$}

It is interesting to note that the very same total derivative term chosen in (5.1) allows us to rewrite $K_{25}$ as

$$
5\left\langle\left(D \gamma_{m n p} A^{1}\right)\left[\left(\lambda \gamma^{m} W^{2}\right)\left(k^{2} \cdot A^{5}\right)-\frac{1}{4}\left(\lambda \gamma^{m} \gamma^{r s} W^{5}\right) \mathcal{F}_{r s}^{2}\right]\left(\lambda \gamma^{n} W^{3}\right)\left(\lambda \gamma^{p} W^{4}\right)\right\rangle-(2 \leftrightarrow 5)
$$

To see this one can use the same kind of manipulations shown in Appendix B to obtain

$$
\begin{gathered}
\left\langle\left(D \gamma_{m n p} A^{1}\right)\left(\lambda \gamma^{m} W^{2}\right)\left(\lambda \gamma^{n} W^{3}\right)\left(\lambda \gamma^{p} W^{4}\right)\left(k^{2} \cdot A^{5}\right)\right\rangle-(2 \leftrightarrow 5)= \\
-8\left\langle\left(\lambda A^{1}\right)\left(\lambda \gamma^{m} W^{2}\right)\left(\lambda \gamma^{n} W^{4}\right) \mathcal{F}_{m n}^{3}\left(k^{2} \cdot A^{5}\right)\right\rangle+4 k_{r}^{2}\left\langle\left(\lambda \gamma^{r} W^{5}\right)\left(\lambda \gamma^{m} W^{2}\right)\left(\lambda \gamma^{n} W^{4}\right)\left(A^{1} \gamma_{m n} W^{3}\right)\right\rangle \\
+4\left(k^{2} \cdot k^{5}\right)\left\langle\left(\lambda A^{5}\right)\left(\lambda \gamma^{m} W^{2}\right)\left(\lambda \gamma^{n} W^{4}\right)\left(A^{1} \gamma_{m n} W^{3}\right)\right\rangle-(2 \leftrightarrow 5)
\end{gathered}
$$

15 Some results in this direction were derived in [32], where it was shown that the four-point kinematic factor can be rewritten as $\left\langle\left(D \gamma_{m n p} A\right)\left(\lambda \gamma^{m} W\right)\left(\lambda \gamma^{n} W\right)\left(\lambda \gamma^{p} W\right)\right\rangle$. 
and

$$
\begin{gathered}
\left\langle\left(D \gamma_{m n p} A^{1}\right)\left(\lambda \gamma^{m} W^{3}\right)\left(\lambda \gamma^{n} W^{4}\right)\left(\lambda \gamma^{p} \gamma^{r s} W^{5}\right) \mathcal{F}_{r s}^{2}\right\rangle-(2 \leftrightarrow 5)= \\
=-8\left\langle\left(\lambda A^{1}\right)\left(\lambda \gamma^{m} \gamma^{r s} W^{5}\right)\left(\lambda \gamma^{n} W^{4}\right) \mathcal{F}_{r s}^{2} \mathcal{F}_{m n}^{3}\right\rangle \\
+16 k_{r}^{2}\left\langle\left(\lambda \gamma^{r} W^{5}\right)\left(\lambda \gamma^{m} W^{2}\right)\left(\lambda \gamma^{n} W^{4}\right)\left(A^{1} \gamma_{m n} W^{3}\right)\right\rangle-(2 \leftrightarrow 5) .
\end{gathered}
$$

Combining (5.6) and (5.7) we get

$$
\begin{gathered}
5\left\langle\left(D \gamma_{m n p} A^{1}\right)\left(\lambda \gamma^{m} W^{2}\right)\left(\lambda \gamma^{n} W^{3}\right)\left(\lambda \gamma^{p} W^{4}\right)\left(k^{2} \cdot A^{5}\right)\right\rangle \\
-\frac{5}{4}\left\langle\left(D \gamma_{m n p} A^{1}\right)\left(\lambda \gamma^{m} W^{3}\right)\left(\lambda \gamma^{n} W^{4}\right)\left(\lambda \gamma^{p} \gamma^{r s} W^{5}\right) \mathcal{F}_{r s}^{2}\right\rangle-(2 \leftrightarrow 5)= \\
=-40\left\langle\left(\lambda A^{1}\right)\left(\lambda \gamma^{m} W^{2}\right)\left(\lambda \gamma^{n} W^{4}\right) \mathcal{F}_{m n}^{3}\left(k^{2} \cdot A^{5}\right)\right\rangle+10\left\langle\left(\lambda A^{1}\right)\left(\lambda \gamma^{m} \gamma^{r s} W^{5}\right)\left(\lambda \gamma^{n} W^{4}\right) \mathcal{F}_{r s}^{2} \mathcal{F}_{m n}^{3}\right\rangle \\
+20\left(k^{2} \cdot k^{5}\right)\left\langle\left(\lambda A^{5}\right)\left(\lambda \gamma^{m} W^{2}\right)\left(\lambda \gamma^{n} W^{4}\right)\left(A^{1} \gamma_{m n} W^{3}\right)\right\rangle-(2 \leftrightarrow 5),
\end{gathered}
$$

which is equal to (5.1), as we wanted to show.

Therefore we have just shown that up to total derivative terms (5.5) is an equivalent answer for the massless five-point kinematic factor at one-loop, which is equal to the result obtained by Lee \& Siegel in 28].

We can also add total derivative terms to $L_{1 j}$, for example

$$
\begin{aligned}
L_{12}=- & 40\left[\left\langle\left[\left(\lambda A^{1}\right)\left(k^{1} \cdot A^{2}\right)+A_{p}^{1}\left(\lambda \gamma^{p} W^{2}\right)\right]\left(\lambda \gamma^{m} W^{5}\right)\left(\lambda \gamma^{n} W^{3}\right) \mathcal{F}_{m n}^{4}\right\rangle\right. \\
+ & 20\left(k^{1} \cdot k^{2}\right)\left\langle\left(\lambda A^{2}\right)\left(\lambda \gamma^{m} W^{5}\right)\left(\lambda \gamma^{n} W^{3}\right)\left(A^{1} \gamma_{m n} W^{4}\right)\right\rangle
\end{aligned}
$$

and one can easily see that these total derivative terms cancel each other out in (4.6). The bosonic components of (5.8) can again be conveniently written as

$$
\begin{aligned}
L_{12}^{5 b}=\frac{1}{72}[ & \left(k^{1} \cdot e^{2}\right) t_{8}^{e^{1}\left(k^{1}+k^{2}\right) e^{3} k^{3} e^{4} k^{4} e^{5} k^{5}}-\left(k^{2} \cdot e^{1}\right) t_{8}^{e^{2}\left(k^{1}+k^{2}\right) e^{3} k^{3} e^{4} k^{4} e^{5} k^{5}} \\
& -\left(e^{1} \cdot e^{2}\right) t_{8}^{k^{1} k^{2} e^{3} k^{3} e^{4} k^{4} e^{5} k^{5}}-\left(k^{1} \cdot k^{2}\right) t_{8}^{\left.e^{1} e^{2} e^{3} k^{3} e^{4} k^{4} e^{5} k^{5}\right]},
\end{aligned}
$$

which is the same as Richards' kinematic factor $A_{12}$.

The expression (5.9) also appears in the analysis of the low-energy limit as equation (4.14) of 39] (see also detailed computations in 40]). There it was shown that $L_{12} /\left(k^{1} \cdot k^{2}\right)$ is the "s-channel" part of the two possible field theory diagrams: when multiplied by $1 /\left(k^{1} \cdot k^{2}\right)$, the first three terms in (5.9) correspond to a Yang-Mills three-vertex 
connected by a propagator to the one-loop $16 t_{8} F^{4}$ vertex, and the last term is matched by the non-linear five-point expansion of the one-loop $t_{8} F^{4}$ vertex.

The correct factorization of massless poles is also evident in the two-fermion component expression (5.4) and can easily be checked in the bosonic result (5.2). The same is true for the four-fermion terms, where one can for example show that up to terms proportional to $k^{2} \cdot k^{5}$

$$
K_{52}^{4 f 1 b}=-\frac{i}{144}\left[\left(k^{25} \cdot k^{4}\right)\left(\chi^{4} \phi^{1}\left(\not k^{1}+\not k^{4}\right) \phi^{25} \chi^{3}\right)+\left(k^{1} \cdot k^{4}\right)\left(\chi^{4} \phi^{25}\left(\not k^{25}+\not k^{4}\right) \phi^{1} \chi^{3}\right)\right]
$$

which is the form of the four-point one-loop kinematic factor as displayed in [27, with $k^{25}=k^{2}+k^{5}$ and $e_{a}^{25}=\left(\chi^{2} \gamma_{a} \chi^{5}\right)$. We have performed this consistency check for all six inequivalent assignment of zero, two or four fermions to the five external states.

The results above suggest that there are no one-loop $F^{5}$ terms in the one-loop effective action (as one could expect from the results of [29] [41]), but we leave the detailed analysis of these matters for the future.

Acknowledgements: CRM would like to thank Jos Vermaseren for quickly fixing (during holidays) a bug in FORM which was triggered by some pattern matchings used in [34]. He would also like to thank Kiyoung Lee for having clarified some points in [28], David Richards for his questions answered about [29], Stefan Theisen and Marc Magro for discussions, Ricardo Medina for email exchange and especially Nathan Berkovits for several discussions, general guidance and suggestions. CS thanks Louise Dolan and Ashok Das for discussions, and Jos Vermaseren for correspondence regarding the trace evaluation in FORM. CRM acknowledges support by the Deutsch-Israelische Projektkooperation (DIP H52) (and also FAPESP grant 04/13290-8 during the early stages of this work). The work of CS was supported by the U.S. Department of Energy, grant no. DE-FG-01-06ER06-01, Task A, and grant no. DE-FG-02-91ER40685.

16 The fact that the kinematic structure of the tree-level and one-loop amplitudes are both proportional to $t_{8} F^{4}$ makes the tree-level discussion of formulae (4.14) and (E.1) of [39] apply equally to our one-loop case. 


\section{Appendix A. Covariant evaluation of the four-point kinematic factor}

In [22] the NMPS kinematic factor of the massless four-point amplitude was shown to be

$$
K_{4}=\left\langle\left(\bar{\lambda} \gamma_{m n p} D\right)\left[\left(\lambda A^{1}\right)\left(\lambda \gamma^{m} W^{2}\right)\left(\lambda \gamma^{n} W^{3}\right)\left(\lambda \gamma^{p} W^{4}\right)\right]\right\rangle
$$

and a proof of equivalence with the minimal pure spinor formalism was presented. That proof however relied on a U(5) decomposition of variables and ignored the overall coefficient relating the two versions. A covariant proof [32] which overcomes these details will now be presented.

Using the SYM equations of motions and the pure spinor constraint it is straightforward to show that

$$
\begin{gathered}
\left(\bar{\lambda} \gamma_{m n p} D\right)\left[\left(\lambda A^{1}\right)\left(\lambda \gamma^{m} W^{2}\right)\left(\lambda \gamma^{n} W^{3}\right)\left(\lambda \gamma^{p} W^{4}\right)\right] \\
=\left[\left(\bar{\lambda} \gamma_{m n p} D\right)\left(\lambda A^{1}\right)\right]\left(\lambda \gamma^{m} W^{2}\right)\left(\lambda \gamma^{n} W^{3}\right)\left(\lambda \gamma^{p} W^{4}\right) \\
+12(\lambda \bar{\lambda})\left(\lambda A^{1}\right)\left[\left(\lambda \gamma^{m} W^{4}\right)\left(\lambda \gamma^{n} W^{2}\right) \mathcal{F}_{m n}^{3}+\left(\lambda \gamma^{m} W^{2}\right)\left(\lambda \gamma^{n} W^{3}\right) \mathcal{F}_{m n}^{4}+\left(\lambda \gamma^{m} W^{3}\right)\left(\lambda \gamma^{n} W^{4}\right) \mathcal{F}_{m n}^{2}\right]
\end{gathered}
$$

Using $\eta_{m n} \gamma_{\alpha(\beta}^{m} \gamma_{\gamma \delta)}^{n}=0$ and that the factor of $\left[\left(\bar{\lambda} \gamma_{m n p} D\right)\left(\lambda A^{1}\right)\right]$ can be substituted by $\left[\left(\bar{\lambda} \gamma_{m} \gamma_{n} \gamma_{p} D\right)\left(\lambda A^{1}\right)\right]$ due to the pure spinor constraint we arrive at the following identity

$$
\begin{aligned}
& \left\langle\left[\left(\bar{\lambda} \gamma_{m n p} D\right)\left(\lambda A^{1}\right)\right]\left(\lambda \gamma^{m} W^{2}\right)\left(\lambda \gamma^{n} W^{3}\right)\left(\lambda \gamma^{p} W^{4}\right)\right\rangle \\
= & \left\langle\left(\bar{\lambda} \gamma_{m} \gamma_{n} W^{3}\right)\left[\left(\lambda \gamma^{n} \gamma_{p} D\right)\left(\lambda A^{1}\right)\right]\left(\lambda \gamma^{m} W^{2}\right)\left(\lambda \gamma^{p} W^{4}\right)\right\rangle \\
+ & \left\langle\left(\bar{\lambda} \gamma_{m} \gamma_{n} \lambda\right)\left(W^{3} \gamma^{n} \gamma_{p}\right)^{\sigma}\left[D_{\sigma}\left(\lambda A^{1}\right)\right]\left(\lambda \gamma^{m} W^{2}\right)\left(\lambda \gamma^{p} W^{4}\right)\right\rangle .
\end{aligned}
$$

Using $\gamma^{n} \gamma_{p}=-\gamma_{p} \gamma^{n}+2 \delta_{p}^{n}$ and the equation of motion $Q(\lambda A)=0$ the first term of (A.2) vanishes, while the second becomes

$$
\begin{gathered}
\left\langle\left(\bar{\lambda} \gamma_{m} \gamma_{n} \lambda\right)\left(W^{3} \gamma^{n} \gamma_{p}\right)^{\sigma}\left[D_{\sigma}\left(\lambda A^{1}\right)\right]\left(\lambda \gamma^{m} W^{2}\right)\left(\lambda \gamma^{p} W^{4}\right)\right\rangle \\
=-2(\lambda \bar{\lambda})\left\langle\left(W^{3} \gamma_{m} \gamma_{p}\right)^{\sigma}\left[(\lambda D) A_{\sigma}^{1}\right]\left(\lambda \gamma^{m} W^{2}\right)\left(\lambda \gamma^{p} W^{4}\right)\right\rangle \\
=+4(\lambda \bar{\lambda})\left\langle\left(\lambda A^{1}\right)\left(\lambda \gamma^{m} W^{2}\right)\left(\lambda \gamma^{n} W^{4}\right) \mathcal{F}_{m n}^{3}\right\rangle,
\end{gathered}
$$

where we used $D_{\alpha} A_{\beta}+D_{\beta} A_{\alpha}=\gamma_{\alpha \beta}^{m} A_{m}$ and integrated the BRST-charge by parts. Therefore

$$
K_{4}=\left\langle\left(\bar{\lambda} \gamma_{m n p} D\right)\left[\left(\lambda A^{1}\right)\left(\lambda \gamma^{m} W^{2}\right)\left(\lambda \gamma^{n} W^{3}\right)\left(\lambda \gamma^{p} W^{4}\right)\right]\right\rangle
$$




$$
\begin{gathered}
=+16(\lambda \bar{\lambda})\left\langle\left(\lambda A^{1}\right)\left(\lambda \gamma^{m} W^{4}\right)\left(\lambda \gamma^{n} W^{2}\right) \mathcal{F}_{m n}^{3}\right\rangle \\
+12(\lambda \bar{\lambda})\left\langle\left(\lambda A^{1}\right)\left(\lambda \gamma^{m} W^{2}\right)\left(\lambda \gamma^{n} W^{3}\right) \mathcal{F}_{m n}^{4}\right\rangle+12(\lambda \bar{\lambda})\left\langle\left(\lambda A^{1}\right)\left(\lambda \gamma^{m} W^{3}\right)\left(\lambda \gamma^{n} W^{4}\right) \mathcal{F}_{m n}^{2}\right\rangle .
\end{gathered}
$$

Furthermore, as was shown in [20], all three correlators above are symmetric in (234), and we obtain

$$
K_{4}=+40(\lambda \bar{\lambda})\left\langle\left(\lambda A^{1}\right)\left(\lambda \gamma^{m} W^{2}\right)\left(\lambda \gamma^{n} W^{3}\right) \mathcal{F}_{m n}^{4}\right\rangle,
$$

which is the covariant proof of equivalence we were looking for.

\section{Appendix B. The computation of the kinematic factor $K_{52}$}

To prove that (3.5) is equal to (3.7) first one uses the SYM equations of motion and the pure spinor constraint to obtain,

$$
\begin{gathered}
\left\langle\left(\bar{\lambda} \gamma_{m n p} D\right)\left[\left(\lambda A^{1}\right) \mathcal{F}_{r s}^{2}\left(\lambda \gamma^{m} \gamma^{r s} W^{5}\right)\left(\lambda \gamma^{n} W^{3}\right)\left(\lambda \gamma^{p} W^{4}\right)\right]\right\rangle \\
=\left\langle\left(\bar{\lambda} \gamma_{m n p} D\right)\left[\left(\lambda A^{1}\right) \mathcal{F}_{r s}^{2}\right]\left(\lambda \gamma^{m} \gamma^{r s} W^{5}\right)\left(\lambda \gamma^{n} W^{3}\right)\left(\lambda \gamma^{p} W^{4}\right)\right\rangle \\
-48(\lambda \bar{\lambda})\left\langle\left(\lambda A^{1}\right)\left(\lambda \gamma^{[m} W^{3}\right)\left(\lambda \gamma^{n]} W^{4}\right) \mathcal{F}_{m u}^{2} \mathcal{F}_{n u}^{5}\right\rangle \\
+12(\lambda \bar{\lambda})\left\langle\left(\lambda A^{1}\right)\left(\lambda \gamma^{m} \gamma^{r s} W^{5}\right) \mathcal{F}_{r s}^{2}\left[\left(\lambda \gamma^{n} W^{4}\right) \mathcal{F}_{m n}^{3}+\left(\lambda \gamma^{n} W^{3}\right) \mathcal{F}_{m n}^{4}\right]\right\rangle \\
+4\left\langle\left(\lambda \gamma^{r s} \bar{\lambda}\right)\left(\lambda A^{1}\right)\left(\lambda \gamma^{m} W^{3}\right)\left(\lambda \gamma^{n} W^{4}\right)\left[\mathcal{F}_{r s}^{2} \mathcal{F}_{m n}^{5}+\mathcal{F}_{r s}^{5} \mathcal{F}_{m n}^{2}\right]\right\rangle \\
-16\left\langle\left(\lambda \gamma^{t u} \bar{\lambda}\right)\left(\lambda A^{1}\right)\left(\lambda \gamma^{[m} W^{3}\right)\left(\lambda \gamma^{n]} W^{4}\right) \mathcal{F}_{m t}^{2} \mathcal{F}_{n u}^{5}\right\rangle .
\end{gathered}
$$

The last two lines vanish after antisymmetrization over [25], therefore we obtain

$$
\begin{gathered}
\left\langle\left(\bar{\lambda} \gamma_{m n p} D\right)\left[\left(\lambda A^{1}\right) \mathcal{F}_{r s}^{2}\left(\lambda \gamma^{m} \gamma^{r s} W^{5}\right)\left(\lambda \gamma^{n} W^{3}\right)\left(\lambda \gamma^{p} W^{4}\right)\right]\right\rangle-(2 \leftrightarrow 5) \\
=\left\langle\left(\bar{\lambda} \gamma_{m n p} D\right)\left[\left(\lambda A^{1}\right) \mathcal{F}_{r s}^{2}\right]\left(\lambda \gamma^{m} \gamma^{r s} W^{5}\right)\left(\lambda \gamma^{n} W^{3}\right)\left(\lambda \gamma^{p} W^{4}\right)\right\rangle \\
+12(\lambda \bar{\lambda})\left\langle\left(\lambda A^{1}\right)\left(\lambda \gamma^{m} \gamma^{r s} W^{5}\right) \mathcal{F}_{r s}^{2}\left[\left(\lambda \gamma^{n} W^{4}\right) \mathcal{F}_{m n}^{3}+\left(\lambda \gamma^{n} W^{3}\right) \mathcal{F}_{m n}^{4}\right]\right\rangle \\
-48(\lambda \bar{\lambda})\left\langle\left(\lambda A^{1}\right)\left(\lambda \gamma^{[m} W^{3}\right)\left(\lambda \gamma^{n]} W^{4}\right) \mathcal{F}_{m u}^{2} \mathcal{F}_{n u}^{5}\right\rangle-(2 \leftrightarrow 5) .
\end{gathered}
$$

The first line of $(\mathbb{B . 1})$ can be rewritten using $\gamma_{\alpha(\beta}^{n}\left(\gamma_{n}\right)_{\gamma \delta)}=0,\left\{\gamma^{m}, \gamma^{n}\right\}=2 \eta^{m n}$ and $\left(\lambda \gamma^{m}\right)_{\alpha}\left(\lambda \gamma_{m}\right)_{\beta}=0$,

$$
\begin{aligned}
& \left(\bar{\lambda} \gamma_{m n p} D\right)\left[\left(\lambda A^{1}\right) \mathcal{F}_{r s}^{2}\right]\left(\lambda \gamma^{m} \gamma^{r s} W^{5}\right)\left(\lambda \gamma^{n} W^{3}\right)\left(\lambda \gamma^{p} W^{4}\right) \\
= & \left(\lambda \gamma_{n} \gamma^{p} D\right)\left[\left(\lambda A^{1}\right) \mathcal{F}_{r s}^{2}\right]\left(\bar{\lambda} \gamma^{m} \gamma^{n} W^{3}\right)\left(\lambda \gamma^{m} \gamma^{r s} W^{5}\right)\left(\lambda \gamma^{p} W^{4}\right)
\end{aligned}
$$




$$
\begin{gathered}
+\left(\bar{\lambda} \gamma_{m} \gamma^{n} \lambda\right)\left(W^{3} \gamma^{n} \gamma^{p} D\right)\left[\left(\lambda A^{1}\right) \mathcal{F}_{r s}^{2}\right]\left(\lambda \gamma^{m} \gamma^{r s} W^{5}\right)\left(\lambda \gamma_{p} W^{4}\right) \\
=-8 k_{r}^{2}\left\langle\left(\lambda A^{1}\right)\left(\lambda \gamma^{m} W^{2}\right)\left(\lambda \gamma^{r} W^{5}\right)\left(\bar{\lambda} \gamma_{m n} W^{3}\right)\left(\lambda \gamma^{n} W^{4}\right)\right\rangle \\
+2(\lambda \bar{\lambda})\left(W^{3} \gamma_{m n} D\right)\left[\left(\lambda A^{1}\right) \mathcal{F}_{r s}^{2}\right]\left(\lambda \gamma^{m} \gamma^{r s} W^{5}\right)\left(\lambda \gamma^{n} W^{4}\right)
\end{gathered}
$$

where we used the equation of motion $(\lambda D)\left(\lambda A^{1}\right)=0$ and a few gamma matrix identities to get

$$
\begin{aligned}
& 2\left\langle(\lambda D)\left[\left(\lambda A^{1}\right) \mathcal{F}_{r s}^{2}\right]\left(\bar{\lambda} \gamma^{m} \gamma^{n} W^{3}\right)\left(\lambda \gamma^{m} \gamma^{r s} W^{5}\right)\left(\lambda \gamma^{n} W^{4}\right)\right\rangle \\
& =-8 k_{r}^{2}\left\langle\left(\lambda A^{1}\right)\left(\lambda \gamma^{m} W^{2}\right)\left(\lambda \gamma^{r} W^{5}\right)\left(\bar{\lambda} \gamma_{m n} W^{3}\right)\left(\lambda \gamma^{n} W^{4}\right)\right\rangle
\end{aligned}
$$

Furthermore, the last term of $(\mathbb{B . 2})$ can be rewritten using $D_{\alpha}\left(\lambda A^{1}\right)=-(\lambda D) A^{1}+$ $\left(\lambda \gamma^{q}\right)_{\alpha} A_{q}$ together with the equations of motion of the SYM superfields,

$$
\begin{aligned}
& 2(\lambda \bar{\lambda})\left(W^{3} \gamma_{m} \gamma_{p} D\right)\left[\left(\lambda A^{1}\right) \mathcal{F}_{r s}^{2}\right]\left(\lambda \gamma^{m} \gamma^{r s} W^{5}\right)\left(\lambda \gamma^{p} W^{4}\right) \\
= & -2(\lambda \bar{\lambda})\left(W^{3} \gamma_{m} \gamma_{p}\right)^{\alpha}\left(Q A_{\alpha}^{1}\right) \mathcal{F}_{r s}^{2}\left(\lambda \gamma^{m} \gamma^{r s} W^{5}\right)\left(\lambda \gamma^{p} W^{4}\right) \\
& +2(\lambda \bar{\lambda}) A_{q}^{1}\left(W^{3} \gamma^{m} \gamma^{p} \gamma^{q} \lambda\right) \mathcal{F}_{r s}^{2}\left(\lambda \gamma^{m} \gamma^{r s} W^{5}\right)\left(\lambda \gamma^{p} W^{4}\right) \\
+ & 4 k_{r}^{2}(\lambda \bar{\lambda})\left(\lambda A^{1}\right)\left(\lambda \gamma^{m} \gamma^{r s} W^{5}\right)\left(\lambda \gamma^{p} W^{4}\right)\left(W^{3} \gamma^{m} \gamma^{p} \gamma^{s} W^{2}\right) .
\end{aligned}
$$

The second line is zero due to the pure spinor condition. Integrating the BRST-charge by parts (B.3) becomes

$$
\begin{aligned}
= & -\frac{1}{2}(\lambda \bar{\lambda})\left\langle\left(\lambda \gamma^{t u} \gamma^{m} \gamma^{p} A^{1}\right) F_{t u}^{3} F_{r s}^{2}\left(\lambda \gamma^{m} \gamma^{r s} W^{5}\right)\left(\lambda \gamma^{p} W^{4}\right)\right\rangle \\
& +\frac{1}{2}(\lambda \bar{\lambda})\left\langle\left(W^{3} \gamma_{m} \gamma_{p} A^{1}\right)\left(\lambda \gamma^{m r s t u} \lambda\right)\left(\lambda \gamma^{p} W^{4}\right) \mathcal{F}_{r s}^{2} \mathcal{F}_{t u}^{5}\right\rangle \\
& +8 k_{r}^{2}(\lambda \bar{\lambda})\left\langle\left(W^{3} \gamma_{m} \gamma_{p} A^{1}\right)\left(\lambda \gamma^{m} W^{2}\right)\left(\lambda \gamma^{r} W^{5}\right)\left(\lambda \gamma^{p} W^{4}\right)\right\rangle \\
+ & 4 k_{r}^{2}(\lambda \bar{\lambda})\left\langle\left(\lambda A^{1}\right)\left(\lambda \gamma^{m} \gamma^{r s} W^{5}\right)\left(\lambda \gamma^{p} W^{4}\right)\left(W^{3} \gamma^{m} \gamma^{p} \gamma^{s} W^{2}\right)\right\rangle .
\end{aligned}
$$

The term (B.4) is equal to $4(\lambda \bar{\lambda})\left(\lambda A^{1}\right)\left(\lambda \gamma^{m} \gamma^{r s} W^{5}\right)\left(\lambda \gamma^{n} W^{4}\right) \mathcal{F}_{r s}^{2} \mathcal{F}_{m n}^{3}$, while (B.5) vanishes after antisymmetrization in [25]. Therefore, putting all of the above together we get

$$
\begin{gathered}
\left\langle\left(\bar{\lambda} \gamma_{m n p} D\right)\left[\left(\lambda A^{1}\right) \mathcal{F}_{r s}^{2}\left(\lambda \gamma^{m} \gamma^{r s} W^{5}\right)\left(\lambda \gamma^{n} W^{3}\right)\left(\lambda \gamma^{p} W^{4}\right)\right]\right\rangle-(2 \leftrightarrow 5)= \\
-8 k_{r}^{2}\left\langle\left(\lambda A^{1}\right)\left(\lambda \gamma^{r} W^{5}\right)\left(\lambda \gamma^{m} W^{2}\right)\left(\lambda \gamma^{n} W^{4}\right)\left(\bar{\lambda} \gamma_{m n} W^{3}\right)\right\rangle \\
-8 k_{r}^{2}(\lambda \bar{\lambda})\left\langle\left(\lambda \gamma^{r} W^{5}\right)\left(\lambda \gamma^{m} W^{2}\right)\left(\lambda \gamma^{n} W^{4}\right)\left(A^{1} \gamma_{m n} W^{3}\right)\right\rangle
\end{gathered}
$$




$$
\begin{gathered}
+4 k_{r}^{2}(\lambda \bar{\lambda})\left\langle\left(\lambda A^{1}\right)\left(\lambda \gamma^{m} \gamma^{r s} W^{5}\right)\left(\lambda \gamma^{n} W^{4}\right)\left(W^{2} \gamma_{s} \gamma_{m n} W^{3}\right)\right\rangle \\
-48(\lambda \bar{\lambda})\left\langle\left(\lambda A^{1}\right)\left(\lambda \gamma^{[m} W^{3}\right)\left(\lambda \gamma^{n]} W^{4}\right) \mathcal{F}_{m u}^{2} \mathcal{F}_{n u}^{5}\right\rangle \\
+12(\lambda \bar{\lambda})\left\langle\left(\lambda A^{1}\right)\left(\lambda \gamma^{m} \gamma^{r s} W^{5}\right)\left(\lambda \gamma^{n} W^{3}\right) \mathcal{F}_{r s}^{2} \mathcal{F}_{m n}^{4}\right\rangle \\
+16(\lambda \bar{\lambda})\left\langle\left(\lambda A^{1}\right)\left(\lambda \gamma^{m} \gamma^{r s} W^{5}\right)\left(\lambda \gamma^{n} W^{4}\right) \mathcal{F}_{r s}^{2} \mathcal{F}_{m n}^{3}\right\rangle-(2 \leftrightarrow 5) .
\end{gathered}
$$

From (A.1) we can also infer that,

$$
\begin{gathered}
\left(\bar{\lambda} \gamma_{m n p} D\right)\left[\left(\lambda A^{1}\right)\left(k^{2} \cdot A^{5}\right)\left(\lambda \gamma^{m} W^{2}\right)\left(\lambda \gamma^{n} W^{3}\right)\left(\lambda \gamma^{p} W^{4}\right)\right]= \\
\left(\bar{\lambda} \gamma_{m n p} D\right)\left[\left(\lambda A^{1}\right)\left(k^{2} \cdot A^{5}\right)\right]\left(\lambda \gamma^{m} W^{2}\right)\left(\lambda \gamma^{n} W^{3}\right)\left(\lambda \gamma^{p} W^{4}\right) \\
+12(\lambda \bar{\lambda})\left(\lambda A^{1}\right)\left(k^{2} \cdot A^{5}\right)\left[\left(\lambda \gamma^{m} W^{4}\right)\left(\lambda \gamma^{n} W^{2}\right) \mathcal{F}_{m n}^{3}+\left(\lambda \gamma^{m} W^{2}\right)\left(\lambda \gamma^{n} W^{3}\right) \mathcal{F}_{m n}^{4}\right. \\
\left.+\left(\lambda \gamma^{m} W^{3}\right)\left(\lambda \gamma^{n} W^{4}\right) \mathcal{F}_{m n}^{2}\right]
\end{gathered}
$$

and repeating similar steps used to prove $(\mathbb{B} .2)$ one gets

$$
\begin{gathered}
\left(\bar{\lambda} \gamma_{m n p} D\right)\left[\left(\lambda A^{1}\right)\left(k^{2} \cdot A^{5}\right)\right]\left(\lambda \gamma^{m} W^{2}\right)\left(\lambda \gamma^{n} W^{3}\right)\left(\lambda \gamma^{p} W^{4}\right)= \\
=-2 k_{r}^{2}\left(\lambda A^{1}\right)\left(\lambda \gamma^{r} W^{5}\right)\left(\lambda \gamma^{m} W^{2}\right)\left(\lambda \gamma^{n} W^{4}\right)\left(\bar{\lambda} \gamma_{m n} W^{3}\right) \\
-2\left(k^{2} \cdot k^{5}\right)\left(\lambda A^{1}\right)\left(\lambda A^{5}\right)\left(\bar{\lambda} \gamma_{m n} W^{3}\right)\left(\lambda \gamma^{m} W^{2}\right)\left(\lambda \gamma^{n} W^{4}\right) \\
+2(\lambda \bar{\lambda})\left(W^{3} \gamma_{m n} D\right)\left[\left(\lambda A^{1}\right)\left(k^{2} \cdot A^{5}\right)\right]\left(\lambda \gamma^{m} W^{2}\right)\left(\lambda \gamma^{n} W^{4}\right)
\end{gathered}
$$

and after several manipulations,

$$
\begin{gathered}
2(\lambda \bar{\lambda})\left(W^{3} \gamma_{m n} D\right)\left[\left(\lambda A^{1}\right)\left(k^{2} \cdot A^{5}\right)\right]\left(\lambda \gamma^{m} W^{2}\right)\left(\lambda \gamma^{n} W^{4}\right) \\
=+4(\lambda \bar{\lambda})\left(\lambda A^{1}\right)\left(\lambda \gamma^{m} W^{2}\right)\left(\lambda \gamma^{n} W^{4}\right) \mathcal{F}_{m n}^{3}\left(k^{2} \cdot A^{5}\right) \\
+2(\lambda \bar{\lambda}) k_{r}^{2}\left(\lambda \gamma^{m} W^{2}\right)\left(\lambda \gamma^{n} W^{4}\right)\left[\left(\lambda A^{1}\right)\left(W^{5} \gamma^{r} \gamma_{m n} W^{3}\right)-\left(\lambda \gamma^{r} W^{5}\right)\left(A^{1} \gamma_{m n} W^{3}\right)\right] \\
+2(\lambda \bar{\lambda})\left(k^{2} \cdot k^{5}\right)\left(\lambda \gamma^{m} W^{2}\right)\left(\lambda \gamma^{n} W^{4}\right)\left[\left(\lambda A^{1}\right)\left(A^{5} \gamma_{m n} W^{3}\right)-\left(\lambda A^{5}\right)\left(A^{1} \gamma_{m n} W^{3}\right)\right]
\end{gathered}
$$

And therefore,

$$
\begin{gathered}
\left(\bar{\lambda} \gamma_{m n p} D\right)\left[\left(\lambda A^{1}\right)\left(k^{2} \cdot A^{5}\right)\left(\lambda \gamma^{m} W^{2}\right)\left(\lambda \gamma^{n} W^{3}\right)\left(\lambda \gamma^{p} W^{4}\right)\right]-(2 \leftrightarrow 5)= \\
=+2(\lambda \bar{\lambda}) k_{r}^{2}\left(\lambda A^{1}\right)\left(\lambda \gamma^{m} W^{2}\right)\left(\lambda \gamma^{n} W^{4}\right)\left(W^{5} \gamma^{r} \gamma_{m n} W^{3}\right) \\
+2 k_{r}^{2}\left(\lambda \gamma^{r} W^{5}\right)\left(\lambda \gamma^{m} W^{2}\right)\left(\lambda \gamma^{n} W^{4}\right)\left[\left(\lambda A^{1}\right)\left(\bar{\lambda} \gamma_{m n} W^{3}\right)-(\lambda \bar{\lambda})\left(A^{1} \gamma_{m n} W^{3}\right)\right]
\end{gathered}
$$




$$
\begin{array}{r}
+12(\lambda \bar{\lambda})\left(\lambda A^{1}\right)\left(k^{2} \cdot A^{5}\right)\left[\left(\lambda \gamma^{m} W^{2}\right)\left(\lambda \gamma^{n} W^{3}\right) \mathcal{F}_{m n}^{4}+\left(\lambda \gamma^{m} W^{3}\right)\left(\lambda \gamma^{n} W^{4}\right) \mathcal{F}_{m n}^{2}\right] \\
+16(\lambda \bar{\lambda})\left(\lambda A^{1}\right)\left(\lambda \gamma^{m} W^{2}\right)\left(\lambda \gamma^{n} W^{4}\right) \mathcal{F}_{m n}^{3}\left(k^{2} \cdot A^{5}\right)-2\left(k^{2} \cdot k^{5}\right) L_{12345}-(2 \leftrightarrow 5),
\end{array}
$$

where we defined

$$
\begin{gathered}
L_{12345}=\left(\lambda A^{1}\right)\left(\lambda A^{5}\right)\left(\lambda \gamma^{m} W^{2}\right)\left(\lambda \gamma^{n} W^{4}\right)\left(\bar{\lambda} \gamma_{m n} W^{3}\right) \\
+(\lambda \bar{\lambda})\left(\lambda A^{5}\right)\left(\lambda \gamma^{m} W^{2}\right)\left(\lambda \gamma^{n} W^{4}\right)\left(A^{1} \gamma_{m n} W^{3}\right)-(\lambda \bar{\lambda})\left(\lambda A^{1}\right)\left(\lambda \gamma^{m} W^{2}\right)\left(\lambda \gamma^{n} W^{4}\right)\left(A^{5} \gamma_{m n} W^{3}\right) .
\end{gathered}
$$

Finally from (B.6) and (B.8) it follows that

$$
\begin{gathered}
K_{52}=\frac{1}{4}\left\langle\left(\bar{\lambda} \gamma_{m n p} D\right)\left[\left(\lambda A^{1}\right) \mathcal{F}_{r s}^{2}\left(\lambda \gamma^{m} \gamma^{r s} W^{5}\right)\left(\lambda \gamma^{n} W^{3}\right)\left(\lambda \gamma^{p} W^{4}\right)\right]\right\rangle \\
-\left\langle\left(\bar{\lambda} \gamma_{m n p} D\right)\left[\left(\lambda A^{1}\right)\left(k^{2} \cdot A^{5}\right)\left(\lambda \gamma^{m} W^{2}\right)\left(\lambda \gamma^{n} W^{3}\right)\left(\lambda \gamma^{p} W^{4}\right)\right]\right\rangle \\
=-16(\lambda \bar{\lambda})\left(\lambda A^{1}\right)\left(\lambda \gamma^{m} W^{2}\right)\left(\lambda \gamma^{n} W^{4}\right) \mathcal{F}_{m n}^{3}\left(k^{2} \cdot A^{5}\right) \\
-12(\lambda \bar{\lambda})\left(\lambda A^{1}\right)\left[\left(\lambda \gamma^{m} W^{2}\right)\left(\lambda \gamma^{n} W^{3}\right) \mathcal{F}_{m n}^{4}+\left(\lambda \gamma^{m} W^{3}\right)\left(\lambda \gamma^{n} W^{4}\right) \mathcal{F}_{m n}^{2}\right]\left(k^{2} \cdot A^{5}\right) \\
+3(\lambda \bar{\lambda})\left(\lambda A^{1}\right)\left(\lambda \gamma^{m} \gamma^{r s} W^{5}\right)\left(\lambda \gamma^{n} W^{3}\right) \mathcal{F}_{r s}^{2} \mathcal{F}_{m n}^{4} \\
+4(\lambda \bar{\lambda})\left(\lambda A^{1}\right)\left(\lambda \gamma^{m} \gamma^{r s} W^{5}\right)\left(\lambda \gamma^{n} W^{4}\right) \mathcal{F}_{r s}^{2} \mathcal{F}_{m n}^{3}, \\
+24(\lambda \bar{\lambda}) k_{r}^{2}\left(\lambda A^{1}\right)\left(\lambda \gamma^{[n} W^{3}\right)\left(\lambda \gamma^{r]} W^{4}\right)\left(W^{5} \gamma^{n} W^{2}\right) \\
-12(\lambda \bar{\lambda})\left(\lambda A^{1}\right)\left(\lambda \gamma^{[m} W^{3}\right)\left(\lambda \gamma^{n]} W^{4}\right) \mathcal{F}_{m u}^{2} \mathcal{F}_{n u}^{5}+2\left(k^{2} \cdot k^{5}\right) L_{12345}-(2 \leftrightarrow 5),
\end{gathered}
$$

where we used $k_{m}^{2}\left(\gamma^{m} W^{2}\right)_{\alpha}=0$ and $k_{r}^{2}\left(\lambda \gamma^{m} \gamma^{r} \gamma^{s} W^{5}\right)\left(W^{2} \gamma^{s} \gamma^{m n} W^{3}\right)=$

$$
=-k_{r}^{2}\left(\lambda \gamma^{m} \gamma^{r} \gamma^{s} \gamma^{m n} W^{3}\right)\left(W^{5} \gamma^{s} W^{2}\right)+k_{r}^{2}\left(\lambda \gamma^{m} \gamma^{r} \gamma^{s} W^{2}\right)\left(W^{5} \gamma^{s} \gamma^{m n} W^{3}\right)
$$

to get

$$
\begin{gathered}
-k_{r}^{2}\left\langle\left(\lambda A^{1}\right)\left(\lambda \gamma^{n} W^{4}\right)\left[\left(\lambda \gamma^{m} \gamma^{r s} W^{5}\right)\left(W^{2} \gamma_{s} \gamma_{m n} W^{3}\right)-2\left(\lambda \gamma^{m} W^{2}\right)\left(W^{5} \gamma^{r} \gamma_{m n} W^{3}\right)\right]\right\rangle \\
=24 k_{r}^{2}\left(\lambda A^{1}\right)\left(\lambda \gamma^{[n} W^{3}\right)\left(\lambda \gamma^{r]} W^{4}\right)\left(W^{5} \gamma^{n} W^{2}\right) .
\end{gathered}
$$

Having obtained $(\overline{B .10})$ the derivation of $(3.7)$ is now finished, as they are equal. 


\section{B.1. Total derivative terms}

We are going to show that $(\mathbb{B} .9)$, when multiplied by $\left(k^{2} \cdot k^{5}\right)$, is part of a total derivative term which vanishes when the whole amplitude is integrated over the position of the vertices. To see this one notes from $(\overline{B .7})$ that those terms come from the evaluation of

$$
\left(\bar{\lambda} \gamma_{m n p} D\right)\left[\left(\lambda A^{1}\right)\left(k^{2} \cdot A^{5}\right)\right]\left(\lambda \gamma^{m} W^{2}\right)\left(\lambda \gamma^{n} W^{3}\right)\left(\lambda \gamma^{p} W^{4}\right)
$$

which is also present in the expressions for $K_{i j}$, where $i, j$ are the same labels as $\left(k^{i} \cdot A^{j}\right)$ in (B.11). Therefore - omitting everything which is not relevant to this proof - the whole amplitude will contain the following terms,

$$
\begin{aligned}
& +L_{15342}\left(k^{1} \cdot k^{2}\right) \eta(2,1)+L_{12543}\left(k^{1} \cdot k^{3}\right) \eta(3,1)+L_{12354}\left(k^{1} \cdot k^{4}\right) \eta(4,1)+L_{12345}\left(k^{1} \cdot k^{5}\right) \eta(5,1) \\
& \quad+\left(k^{2} \cdot k^{3}\right)\left[L_{12543} \eta(3,2)+L_{15342} \eta(2,3)\right]+\left(k^{3} \cdot k^{4}\right)\left[L_{12354} \eta(4,3)+L_{12543} \eta(3,4)\right] \\
& \quad+\left(k^{2} \cdot k^{4}\right)\left[L_{12354} \eta(4,2)+L_{15342} \eta(2,4)\right]+\left(k^{4} \cdot k^{5}\right)\left[L_{12345} \eta(5,4)+L_{12354} \eta(4,5)\right] \\
& +\left(k^{3} \cdot k^{5}\right)\left[L_{12345} \eta(5,3)+L_{12543} \eta(3,5)\right]+\left(k^{2} \cdot k^{5}\right)\left[L_{12345} \eta(5,2)+L_{15342} \eta(2,5)\right] . \quad \text { B.12) }
\end{aligned}
$$

By pairing the above terms in groups of four one can show that they vanish. For example, consider the terms which contain $\eta(5, j)$. It is easy to show that they are a total derivative in the variable $z_{5}$,

$$
\begin{gathered}
+L_{12345}\left[\left(k^{1} \cdot k^{5}\right) \eta(5,1)+\left(k^{2} \cdot k^{5}\right) \eta(5,2)+\left(k^{3} \cdot k^{5}\right) \eta(5,3)+\left(k^{4} \cdot k^{5}\right) \eta(5,4)=\right. \\
=-\frac{\partial}{\partial z_{5}} \exp \left[\sum_{i<j}^{5}\left(k^{i} \cdot k^{j}\right) f\left(z_{i}, z_{j}\right)\right]
\end{gathered}
$$

because $\eta\left(z_{5}, z_{j}\right)=-\frac{\partial}{\partial z_{5}} f\left(z_{j}, z_{5}\right)$. The same can be shown for the remaining terms of $(\mathbb{B} .12)$, establishing that they will all vanish by the cancelled propagator argument.

As can be easily inspected, the symmetry in the labels (34) is manifest in (3.6) but not in $(\overline{B .10})$. Upon subtraction of the $(3 \leftrightarrow 4)$ permutation in $(\mathbb{B . 1 0})$ one arrives at the following identity

$$
\begin{gathered}
\left\langle\left(\lambda A^{1}\right)\left[4\left(\lambda \gamma^{m} W^{2}\right)\left(k^{2} \cdot A^{5}\right)-\left(\lambda \gamma^{m} \gamma^{r s} W^{5}\right) \mathcal{F}_{r s}^{2}\right]\left(\lambda \gamma^{n} W^{4}\right) \mathcal{F}_{m n}^{3}\right\rangle-(2 \leftrightarrow 5) \\
=\left\langle\left(\lambda A^{1}\right)\left[4\left(\lambda \gamma^{m} W^{2}\right)\left(k^{2} \cdot A^{5}\right)-\left(\lambda \gamma^{m} \gamma^{r s} W^{5}\right) \mathcal{F}_{r s}^{2}\right]\left(\lambda \gamma^{n} W^{3}\right) \mathcal{F}_{m n}^{4}\right\rangle \\
+4\left(k^{2} \cdot k^{5}\right)\left(\lambda A^{1}\right)\left(\lambda A^{5}\right)\left(\lambda \gamma^{m} W^{2}\right)\left(W^{3} \gamma_{m} W^{4}\right)
\end{gathered}
$$




$$
\begin{gathered}
+2\left(k^{2} \cdot k^{5}\right)\left(\lambda A^{1}\right)\left(\lambda \gamma^{m} W^{2}\right)\left[\left(\lambda \gamma^{n} W^{3}\right)\left(A^{5} \gamma_{m n} W^{4}\right)-\left(\lambda \gamma^{n} W^{4}\right)\left(A^{5} \gamma_{m n} W^{3}\right)\right] \\
-2\left(k^{2} \cdot k^{5}\right)\left(\lambda A^{5}\right)\left(\lambda \gamma^{m} W^{2}\right)\left[\left(\lambda \gamma^{n} W^{3}\right)\left(A^{1} \gamma_{m n} W^{4}\right)-\left(\lambda \gamma^{n} W^{4}\right)\left(A^{1} \gamma_{m n} W^{3}\right)\right]-(2 \leftrightarrow 5),
\end{gathered}
$$

where all terms which contain an explicit $\left(k^{2} \cdot k^{5}\right)$ factor come from the total derivative terms as shown in the previous paragraph $\sqrt{17}$. The above can be rewritten as

$$
\begin{gathered}
\left\langle\left(\lambda A^{1}\right)\left[4\left(\lambda \gamma^{m} W^{2}\right)\left(k^{2} \cdot A^{5}\right)-\left(\lambda \gamma^{m} \gamma^{r s} W^{5}\right) \mathcal{F}_{r s}^{2}\right]\left(\lambda \gamma^{n} W^{4}\right) \mathcal{F}_{m n}^{3}\right\rangle-(2 \leftrightarrow 5) \\
=\left\langle\left(\lambda A^{1}\right)\left[4\left(\lambda \gamma^{m} W^{2}\right)\left(k^{2} \cdot A^{5}\right)-\left(\lambda \gamma^{m} \gamma^{r s} W^{5}\right) \mathcal{F}_{r s}^{2}\right]\left(\lambda \gamma^{n} W^{3}\right) \mathcal{F}_{m n}^{4}\right\rangle \\
-4\left(k^{2} \cdot k^{5}\right)\left(\lambda A^{1}\right)\left(\lambda A^{5}\right)\left(\lambda \gamma^{m} W^{2}\right)\left(W^{3} \gamma_{m} W^{4}\right)-(2 \leftrightarrow 5)
\end{gathered}
$$

where we used $\left(\lambda \gamma^{m} W^{2}\right)\left[\left(A^{1} \gamma^{m n} W^{4}\right)\left(\lambda \gamma^{n} W^{3}\right)-(3 \leftrightarrow 4)\right]=2\left(\lambda \gamma^{m} W^{2}\right)\left(\lambda A^{1}\right)\left(W^{3} \gamma^{m} W^{4}\right)$.

Furthermore, the relation

$$
\begin{gathered}
Q\left[\left(W^{2} \gamma_{m n p} W^{3}\right)\left(\lambda \gamma^{m n p} W^{4}\right)\right]=24\left(\lambda \gamma^{m} W^{2}\right)\left(\lambda \gamma^{n} W^{3}\right) \mathcal{F}_{m n}^{4} \\
-12\left(\lambda \gamma^{m} W^{2}\right)\left(\lambda \gamma^{n} W^{4}\right) \mathcal{F}_{m n}^{3}-12\left(\lambda \gamma^{m} W^{4}\right)\left(\lambda \gamma^{n} W^{3}\right) \mathcal{F}_{m n}^{2}
\end{gathered}
$$

and the fact that correlations of BRST-trivial operators vanish can be used to show

$$
\begin{gathered}
24\left\langle\left(\lambda A^{1}\right)\left(\lambda \gamma^{m} W^{2}\right)\left(\lambda \gamma^{n} W^{3}\right) \mathcal{F}_{m n}^{4} \int U^{5}\right\rangle= \\
=12\left\langle\left(\lambda A^{1}\right)\left(\lambda \gamma^{m} W^{3}\right)\left(\lambda \gamma^{n} W^{4}\right) \mathcal{F}_{m n}^{2} \int U^{5}\right\rangle+12\left\langle\left(\lambda A^{1}\right)\left(\lambda \gamma^{m} W^{2}\right)\left(\lambda \gamma^{n} W^{4}\right) \mathcal{F}_{m n}^{3} \int U^{5}\right\rangle \\
+\left\langle\left(\lambda A^{1}\right)\left(W^{2} \gamma_{m n p} W^{3}\right)\left(\lambda \gamma^{m n p} W^{4}\right) \int \partial\left(\lambda A^{5}\right)\right\rangle .
\end{gathered}
$$

However care has to be taken when computing the OPEs in (B.14) because of noncommuting operators. For example, when considering the term appearing in the derivation of (B.14), namely $\left\langle\left(\lambda A^{1}\right) Q\left[\left(W^{2} \gamma_{m n p} W^{3}\right)\left(\lambda \gamma^{m n p} W^{4}\right)\right] \int U^{5}\right\rangle$ one can first compute the OPEs and then "integrate" the BRST charge by parts or the other way around; and these two operations don't commute. Using $\partial\left(\lambda A^{5}\right)=\Pi^{m} k_{m}^{5}\left(\lambda A^{5}\right)+\partial \theta^{\alpha} \partial_{\alpha}\left(\lambda A^{5}\right)$ and with the above caveat one can check that the components proportional to $\eta\left(z_{2}, z_{5}\right)$ obey

$-24\left\langle\left(\lambda A^{1}\right)\left(\lambda \gamma^{m} W^{3}\right)\left(\lambda \gamma^{n} W^{4}\right) k_{[m}^{2}\left(W^{5} \gamma_{n]} W^{2}\right)\right\rangle-12\left\langle\left(\lambda A^{1}\right)\left(\lambda \gamma^{m} W^{3}\right)\left(\lambda \gamma^{n} W^{4}\right) \mathcal{F}_{m n}^{2}\left(k^{2} \cdot A^{5}\right)\right\rangle$

17 We nevertheless keep track of them because they are useful when performing component expansion checks with the computer. 


$$
\begin{gathered}
-12\left\langle\left(\lambda A^{1}\right)\left(\lambda \gamma^{[m} W^{3}\right)\left(\lambda \gamma^{n]} W^{4}\right) \mathcal{F}_{m t}^{2} \mathcal{F}_{n t}^{5}\right. \\
=-24\left\langle\left(\lambda A^{1}\right)\left(\lambda \gamma^{m} W^{2}\right)\left(\lambda \gamma^{n} W^{3}\right) \mathcal{F}_{m n}^{4}\left(k^{2} \cdot A^{5}\right)\right\rangle+6\left\langle\left(\lambda A^{1}\right)\left(\lambda \gamma^{m} \gamma^{r s} W^{5}\right)\left(\lambda \gamma^{n} W^{3}\right) \mathcal{F}_{r s}^{2} \mathcal{F}_{m n}^{4}\right\rangle \\
+12\left\langle\left(\lambda A^{1}\right)\left(\lambda \gamma^{m} W^{2}\right)\left(\lambda \gamma^{n} W^{4}\right) \mathcal{F}_{m n}^{3}\left(k^{2} \cdot A^{5}\right)\right\rangle-3\left\langle\left(\lambda A^{1}\right)\left(\lambda \gamma^{m} \gamma^{r s} W^{5}\right)\left(\lambda \gamma^{n} W^{4}\right) \mathcal{F}_{r s}^{2} \mathcal{F}_{m n}^{3}\right\rangle \\
-\left(k^{2} \cdot k^{5}\right)\left\langle\left(\lambda A^{1}\right)\left(\lambda A^{5}\right)\left(W^{2} \gamma_{m n p} W^{3}\right)\left(\lambda \gamma^{m n p} W^{4}\right)\right\rangle-(2 \leftrightarrow 5) .
\end{gathered}
$$

where the third term in the left-hand side is related to the order in which one chooses to compute the OPEs to generate $\eta\left(z_{2}, z_{5}\right)$ or to integrate the BRST charge by parts. It is the result of integrating the BRST charge by parts after computing the OPE $d_{\alpha}\left(z_{5}\right) \theta^{\beta}\left(z_{2}\right)$ as $z_{5} \rightarrow z_{2}$

$$
\begin{aligned}
& -\frac{1}{4}\left(\lambda A^{1}\right) Q\left[F_{r s}^{2}\left(\gamma^{r s} \gamma_{m n p} W^{3}\right)_{\alpha}\left(\lambda \gamma^{m n p} W^{4}\right)\right] W_{5}^{\alpha}-(2 \leftrightarrow 5) \\
& =-12\left\langle\left(\lambda A^{1}\right)\left(\lambda \gamma^{[m} W^{3}\right)\left(\lambda \gamma^{n]} W^{4}\right) \mathcal{F}_{m t}^{2} \mathcal{F}_{n t}^{5} \eta(5,2)-(2 \leftrightarrow 5) .\right.
\end{aligned}
$$

Note also that the term with the explicit $\left(k^{2} \cdot k^{5}\right)$ factor also comes from a total derivative term, and can therefore be dropped in the end 18 .

Substituting ( $\mathrm{B} .15)$ in $(\overline{\mathrm{B} .10})$ and then using (B.13) allows one to obtain the following expression for $K_{52}$

$$
\begin{gathered}
(\lambda \bar{\lambda})^{-1} K_{52}= \\
-40\left\langle\left(\lambda A^{1}\right)\left(\lambda \gamma^{m} W^{2}\right)\left(\lambda \gamma^{n} W^{3}\right) \mathcal{F}_{m n}^{4}\left(k^{2} \cdot A^{5}\right)\right\rangle+10\left\langle\left(\lambda A^{1}\right)\left(\lambda \gamma^{m} \gamma^{r s} W^{5}\right)\left(\lambda \gamma^{n} W^{3}\right) \mathcal{F}_{r s}^{2} \mathcal{F}_{m n}^{4}\right\rangle \\
+\left(k^{2} \cdot k^{5}\right)\left[4\left\langle\left(\lambda A^{1}\right)\left(\lambda A^{5}\right)\left(\lambda \gamma^{m} W^{2}\right)\left(W^{3} \gamma_{m} W^{4}\right)\right\rangle-\left\langle\left(\lambda A^{1}\right)\left(\lambda A^{5}\right)\left(W^{2} \gamma_{m n p} W^{3}\right)\left(\lambda \gamma^{m n p} W^{4}\right)\right\rangle\right] \\
+\left(k^{2} \cdot k^{5}\right) L_{12345}-(2 \leftrightarrow 5),
\end{gathered}
$$

which dropping total derivative terms can be expressed simply as

$$
\begin{gathered}
(\lambda \bar{\lambda})^{-1} K_{52}= \\
-40\left\langle\left(\lambda A^{1}\right)\left(\lambda \gamma^{m} W^{2}\right)\left(\lambda \gamma^{n} W^{3}\right) \mathcal{F}_{m n}^{4}\left(k^{2} \cdot A^{5}\right)\right\rangle+10\left\langle\left(\lambda A^{1}\right)\left(\lambda \gamma^{m} \gamma^{r s} W^{5}\right)\left(\lambda \gamma^{n} W^{3}\right) \mathcal{F}_{r s}^{2} \mathcal{F}_{m n}^{4}\right\rangle \\
-(2 \leftrightarrow 5),
\end{gathered}
$$

therefore concluding the proof of equation (4.1).

18 We chose to keep it to be able to check (B.15) explicitly by a component expansion computation with FORM [33] [34]. 


\section{Appendix C. Integrating $\bar{\lambda}_{\alpha}$ in pure spinor superspace expressions}

In principle one-loop calculations using the PS formalism will involve superspace integrals in which the non-minimal pure spinor $\bar{\lambda}_{\alpha}$ does not simply occur as a product $(\lambda \bar{\lambda})$, and one should know how to deal with these expressions. Although in this paper we managed to isolate this "new" type of correlator to the total derivative terms we will nevertheless show how to compute them explicitly.

As with the $\left\langle\lambda^{3} \theta^{5}\right\rangle$ correlators, the result is completely fixed by symmetry. Writing $S^{ \pm}$for the spinor irreps of $\mathrm{SO}(10)$ and, schematically, $P^{ \pm}$for pure spinors, we find the representation content 42]

$$
\begin{aligned}
& \bar{\lambda}_{\epsilon} \lambda^{(\alpha} \lambda^{\beta} \lambda^{\gamma} \lambda^{\delta)}: S^{-} \otimes \operatorname{Sym}^{4} S^{+}=2 \times[00003] \oplus 1 \times[11010] \oplus \ldots \\
& P^{-} \otimes \operatorname{Sym}^{4} P^{+}=1 \times[00003] \oplus \ldots \\
& \theta^{\left[\delta_{1}\right.} \ldots \theta^{\left.\delta_{5}\right]}: \mathrm{Alt}^{5} S^{+}=1 \times[00030] \oplus 1 \times[11010],
\end{aligned}
$$

so that there is only one invariant combination of $\bar{\lambda} \lambda^{3}$ and $\theta^{5}$. We will now make use of this uniqueness and construct a spinorial formula relating it to the $\left\langle\lambda^{3} \theta^{5}\right\rangle$ case, where

$$
\left\langle\lambda^{\alpha} \lambda^{\beta} \lambda^{\gamma} \theta^{\delta_{1}} \cdots \theta^{\delta_{5}}\right\rangle \equiv \bar{T}^{\alpha \beta \gamma, \delta_{1} \ldots \delta_{5}}=N^{-1}\left[\left(\gamma^{m}\right)^{\alpha \delta_{1}}\left(\gamma^{n}\right)^{\beta \delta_{2}}\left(\gamma^{p}\right)^{\gamma \delta_{3}}\left(\gamma_{m n p}\right)^{\delta_{4} \delta_{5}}\right]_{(\alpha \beta \gamma)\left[\delta_{1} \ldots \delta_{5}\right]},
$$

with $N$ a normalization constant. The simplest ansatz is to write $\left\langle\bar{\lambda} \lambda^{3} \theta^{5}\right\rangle=\delta \times \bar{T}$, suitably symmetrized. However, this time the pure spinor property is essential to the uniqueness argument and we will need to be careful to subtract gamma traces. This can be done by applying the projection operator

$$
\mathcal{P}_{\rho \sigma \tau \omega}^{\alpha \beta \gamma \delta}=\frac{1}{2772}\left[\delta_{\rho}^{(\alpha} \delta_{\sigma}^{\beta} \delta_{\tau}^{\gamma} \gamma \delta_{\omega}^{\delta)}-\frac{1}{4} \delta_{(\rho}^{(\alpha} \delta_{\sigma}^{\beta} \gamma_{\tau \omega)}^{a} \gamma_{a}^{\gamma \delta)}+\frac{1}{160} \gamma_{(\rho \sigma}^{a} \gamma_{a}^{(\alpha \beta} \gamma_{\tau \omega)}^{b} \gamma_{b}^{\gamma \delta)}\right],
$$

which is symmetric and gamma-traceless in both sets of indices and satisfies $\mathcal{P} \mathcal{P}=\mathcal{P}$. We conclude that

$$
\left\langle\bar{\lambda}_{\epsilon} \lambda^{\alpha_{1}} \cdots \lambda^{\alpha_{4}} \theta^{\delta_{1}} \cdots \theta^{\delta_{5}}\right\rangle=c \times \mathcal{P}_{\gamma_{1} \ldots \gamma_{4}}^{\alpha_{1} \ldots \alpha_{4}}\left[\delta_{\epsilon}^{\gamma_{4}} \bar{T}^{\gamma_{1} \gamma_{2} \gamma_{3}, \delta_{i}}\right]
$$

for some constant $c$. Substituting (C.1) and using that $\bar{T}$ is gamma-traceless, we get

$$
\left\langle\bar{\lambda}_{\epsilon} \lambda^{\alpha_{i}} \theta^{\delta_{i}}\right\rangle=\frac{c}{2772}\left[\delta_{\epsilon}^{\left(\alpha_{1}\right.} \bar{T}^{\left.\alpha_{2} \alpha_{3} \alpha_{4}\right), \delta_{i}}-\frac{1}{8} \delta_{\left(\gamma_{1}\right.}^{\left(\alpha_{1}\right.} \delta_{\gamma_{2}}^{\alpha_{2}}\left(\gamma^{m}\right)_{\left.\gamma_{3}\right) \epsilon}\left(\gamma_{m}\right)^{\left.\alpha_{3} \alpha_{4}\right)} \bar{T}^{\gamma_{i}, \delta_{i}}\right]
$$

The normalization is not important for our amplitude calculation, as all our expressions contain one $\bar{\lambda}$ and four $\lambda$ s. We will set

$$
\left\langle(\bar{\lambda} \lambda)\left(\lambda \gamma^{m} \theta\right)\left(\lambda \gamma^{n} \theta\right)\left(\lambda \gamma^{p} \theta\right)\left(\theta \gamma_{m n p} \theta\right)\right\rangle=1
$$


which is satisfied for $c=672$. Equation (C.2) can be interpreted in form of a practical prescription by re-writing it as

$$
\left\langle\bar{\lambda}_{\epsilon} \lambda^{\alpha_{i}} \theta^{\delta_{i}}\right\rangle=\frac{2}{33}(\lambda \bar{\lambda})\left[\left\{\delta_{\epsilon}^{\alpha_{1}}\left\langle\lambda^{\alpha_{2}} \lambda^{\alpha_{3}} \lambda^{\alpha_{4}} \theta^{\delta_{i}}\right\rangle+\cdots\right\}-\frac{1}{12}\left\{\gamma_{m}^{\alpha_{1} \alpha_{2}}\left\langle\lambda^{\alpha_{3}} \lambda^{\alpha_{4}}\left(\lambda \gamma^{m}\right)_{\epsilon} \theta^{\delta_{i}}\right\rangle+\cdots\right\}\right]
$$

where the two curly brackets contain four and twelve terms respectively, corresponding to the choice of $\alpha$ indices on $\delta_{\epsilon}^{\alpha}$ and on $\gamma_{m}^{\alpha_{i} \alpha_{j}}$. We can think of the first group of terms as coming from eliminating the $\bar{\lambda}$ and one of the $\lambda$ s, contracting their indices. In the second group, a pair of $\lambda^{\alpha_{i}} \lambda^{\alpha_{j}}$ has been replaced by $\gamma_{m}^{\alpha_{i} \alpha_{j}}$ and $\bar{\lambda}_{\epsilon}$ by $\left(\lambda \gamma_{m}\right)_{\epsilon}$. We have thus reduced everything to the well-known $\left\langle\lambda^{3} \theta^{5}\right\rangle$ correlators.

For example, applying this procedure to the eighth term in (3.7), we obtain (inside correlators):

$$
\begin{aligned}
& \left(\lambda A^{1}\right)\left(\lambda A^{5}\right)\left(\lambda \gamma^{m} W^{2}\right)\left(\lambda \gamma^{n} W^{4}\right)\left(\bar{\lambda} \gamma_{m n} W^{3}\right)=\frac{2}{33}[ \\
& \left\{-\left(A^{1} \gamma_{m n} W^{3}\right)\left(\lambda A^{5}\right)\left(\lambda \gamma^{m} W^{2}\right)\left(\lambda \gamma^{n} W^{4}\right)+\left(\lambda A^{1}\right)\left(A^{5} \gamma_{m n} W^{3}\right)\right)\left(\lambda \gamma^{m} W^{2}\right)\left(\lambda \gamma^{n} W^{4}\right) \\
& \left.\left.-\left(\lambda A^{1}\right)\left(\lambda A^{5}\right)\left(W^{2} \gamma^{m} \gamma_{m n} W^{3}\right)\right)\left(\lambda \gamma^{n} W^{4}\right)+\left(\lambda A^{1}\right)\left(\lambda A^{5}\right)\left(\lambda \gamma^{m} W^{2}\right)\left(W^{4} \gamma^{n} \gamma_{m n} W^{3}\right)\right\} \\
& -\frac{1}{12}\left\{\left(\left(A^{1} \gamma^{a} A^{5}\right)\left(\lambda \gamma^{m} W^{2}\right)\left(\lambda \gamma^{n} W^{4}\right)-\left(A^{1} \gamma^{a} \gamma^{m} W^{2}\right)\left(\lambda A^{5}\right)\left(\lambda \gamma^{n} W^{4}\right)\right.\right. \\
& \quad+\left(A^{1} \gamma^{a} \gamma^{n} W^{4}\right)\left(\lambda A^{5}\right)\left(\lambda \gamma^{m} W^{2}\right)+\left(\lambda A^{1}\right)\left(A^{5} \gamma^{a} \gamma^{m} W^{2}\right)\left(\lambda \gamma^{n} W^{4}\right) \\
& \left.\left.\left.\quad-\left(\lambda A^{1}\right)\left(A^{5} \gamma^{n} W^{4}\right)\left(\lambda \gamma^{m} W^{2}\right)+\left(\lambda A^{1}\right)\left(\lambda A^{5}\right)\left(W^{2} \gamma^{m} \gamma^{a} W^{4}\right)\right)\left(\lambda \gamma_{a} \gamma_{m n} W^{3}\right)\right\}\right]
\end{aligned}
$$

To check the consistency of (C.5) one can use the identity

$$
\begin{gathered}
\left\langle\left(\lambda A^{1}\right)\left(\lambda A^{5}\right)\left(\lambda \gamma^{m} W^{2}\right)\left[\left(\lambda \gamma^{n} W^{4}\right)\left(\bar{\lambda} \gamma_{m n} W^{3}\right)-(3 \leftrightarrow 4)\right]\right\rangle \\
=2\left\langle(\lambda \bar{\lambda})\left(\lambda A^{1}\right)\left(\lambda A^{5}\right)\left(\lambda \gamma^{m} W^{2}\right)\left(W^{3} \gamma_{m} W^{4}\right)\right\rangle
\end{gathered}
$$

and compute each term of the lhs of (C.6) using the corresponding result of (C.5) and compare it against the direct computation of the rhs using the standard $\left\langle\lambda^{3} \theta^{5}\right\rangle$ correlators. We did this and obtained agreement.

Alternatively, we can follow the methods of [17] and derive tensorial formulae by constructing a symmetry-based ansatz and using pure spinor identities to relate it to the normalization condition (C.3). Proceeding in this fashion, we find

$$
\left\langle\left(\bar{\lambda} \gamma^{a b} \lambda\right)\left(\lambda \gamma^{c} \theta\right)\left(\lambda \gamma^{d} \theta\right)\left(\lambda \gamma^{e} \theta\right)\left(\theta \gamma^{r s t} \theta\right)\right\rangle=\frac{1}{140}\left[\delta_{c r}^{a b} \delta_{s t}^{d e}\right]_{[c d e][r s t]}
$$




$$
\begin{aligned}
& \left\langle\left(\bar{\lambda} \gamma^{a b} \lambda\right)\left(\lambda \gamma^{c d e} \theta\right)\left(\lambda \gamma^{f} \theta\right)\left(\lambda \gamma^{g} \theta\right)\left(\theta \gamma^{r s t} \theta\right)\right\rangle=\frac{1}{4620}\left[\delta_{a b}^{f g} \delta_{c d e}^{r s t}+2 \delta_{c d e}^{a s t} \delta_{b}^{g} \delta_{r}^{f}-20 \delta_{c d e}^{f s t} \delta_{b}^{g} \delta_{r}^{a}\right. \\
& \left.+23 \delta_{c d e}^{a b t} \delta_{r s}^{f g}+24 \delta_{c d e}^{a f t} \delta_{r s}^{b g}+\delta_{c d e}^{f g t} \delta_{r s}^{a b}\right]_{[a b][c d e][f g][r s t]}-\frac{1}{25200} \epsilon^{a b c d e f g r s t} \\
& \left\langle\left(\bar{\lambda} \gamma^{a b} \lambda\right)\left(\lambda \gamma^{c d e} \theta\right)\left(\lambda \gamma^{f g h} \theta\right)\left(\lambda \gamma^{i} \theta\right)\left(\theta \gamma^{r s t} \theta\right)\right\rangle \\
& =\frac{1}{3850}\left[36 \delta_{a b r}^{c d e} \delta_{i s t}^{f g h}-8 \delta_{a i r}^{c d e} \delta_{b s t}^{f g h}+4 \delta_{f a r}^{c d e} \delta_{s t}^{g h} \delta_{b}^{i}-2 \delta_{f a i}^{c d e} \delta_{s t}^{g h} \delta_{b}^{r}-12 \delta_{f a r}^{c d e} \delta_{i s}^{g h} \delta_{b}^{t}\right. \\
& \left.\quad-46 \delta_{f a b}^{c d e} \delta_{r s t}^{g h i}-42 \delta_{f g a}^{c d e} \delta_{r s t}^{b h i}+2 \delta_{f g i}^{c d e} \delta_{r s t}^{a b h}-9 \delta_{f g r}^{c d e} \delta_{s t}^{h b} \delta_{a}^{i}\right]_{[a b][c d e][f g h][r s t][c d e \leftrightarrow f g h]} \\
& \quad+\frac{1}{4200}\left(\delta_{[a}^{[r \mid} \epsilon_{b]}^{c d e f g h i \mid s t]}+\delta_{[c}^{[r \mid} \epsilon^{a b}{ }_{d e]}^{f g h i \mid s t]}-\delta_{[f}^{[r \mid} \epsilon^{a b c d e}{ }_{g h]}{ }_{i s t]}\right) \\
& \left\langle\left(\bar{\lambda} \gamma^{a b c d} \lambda\right)\left(\lambda \gamma^{e} \theta\right)\left(\lambda \gamma^{f} \theta\right)\left(\lambda \gamma^{g} \theta\right)\left(\theta \gamma^{r s t} \theta\right)\right\rangle \\
& =-\frac{1}{70}\left[\delta_{e f r s}^{a b c d} \delta_{g}^{t}\right]_{[e f g][r s t]}+\frac{1}{25200} \epsilon^{a b c d e f g r s t} \\
& \left\langle\left(\bar{\lambda} \gamma^{a b c d} \lambda\right)\left(\lambda \gamma^{e f g} \theta\right)\left(\lambda \gamma^{h} \theta\right)\left(\lambda \gamma^{i} \theta\right)\left(\theta \gamma^{r s t} \theta\right)\right\rangle=\frac{1}{1925}\left[23 \delta_{a b c d}^{e f g r} \delta_{h i}^{s t}-42 \delta_{a b c d}^{e f h r} \delta_{g i}^{s t}\right. \\
& \left.\quad-2 \delta_{a b c d}^{e f r s} \delta_{g}^{h} \delta_{i}^{t}+\delta_{a b c d}^{e h i r} \delta_{f g}^{s t}+20 \delta_{a b c d}^{e h r s} \delta_{f g}^{i t}+\delta_{a b c d}^{e r s t} \delta_{f g}^{h i}\right]_{[a b c d][e f g][h i][r s t]} \\
& \quad \frac{1}{4200}\left(2 \delta_{[r}^{[a} \epsilon^{b c d] e f g h i}{ }_{s t]}+\delta_{[r}^{[e \mid} \epsilon^{a b c d \mid f g] h i}{ }_{s t]}\right)
\end{aligned}
$$

This approach is useful if one aims at a direct component evaluation without being interested in equivalent $\left\langle\lambda^{3} \theta^{5}\right\rangle$ correlators, e.g. when checking superspace manipulations with a computer.

\section{Appendix D. Component evaluation of pure spinor superspace correlators}

While amplitude expressions in pure spinor superspace comprise bosonic and fermionic parts in the form of superfields, it is often necessary to extract separate components, e.g. to compare with existing results. Comparisons of component expansions also provide a valuable check on superspace manipulations. In this appendix, we summarize some techniques and intermediate results relevant to the present paper, extending methods published previously 22] [19].

In all but the simplest cases, the evaluation of pure spinor correlators becomes computationally involved, and the help of a computer algebra system seems indispensable. We therefore emphasize approaches that may be forbidding for a paper-and-pen calculation, but lend themselves to direct transfer to the computer. The present authors have employed independent implementations of the algorithms, using FORM and Mathematica respectively. 


\section{D.1. Superfield expansions}

The fermionic expansions of the Yang-Mills superfields satisfy simple recursion relations, which makes explicit component expressions readily available [43] [25]. In addition, we need the expansion of the antisymmetrised derivative of the spinor gauge superfield, $D_{\alpha} \gamma_{m n p}^{\alpha \beta} A_{\beta}$. This object vanishes at order $\theta^{0}$, and the next few orders can be written as

$$
\begin{aligned}
& \left(D \gamma_{m n p} A\right)^{(1)}=-\frac{4}{3}\left(\theta \gamma_{m n p} \chi\right) \\
& \left(D \gamma_{m n p} A\right)^{(2)}=\frac{1}{4}\left(\theta \gamma_{m n p} \not F\right)+\frac{1}{4} \partial_{r} \zeta_{s}\left(\theta \gamma^{r} \gamma_{m n p} \gamma^{s} \theta\right) \\
& \left(D \gamma_{m n p} A\right)^{(3)}=\left(\theta \gamma_{a[m n} \theta\right)\left(\theta \gamma^{a} \partial_{p]} \chi\right)-\frac{1}{5}\left(\theta \gamma_{[m n}^{a} \theta\right)\left(\theta \gamma_{p]} \partial_{a} \chi\right) \\
& \left(D \gamma_{m n p} A\right)^{(4)}=\frac{1}{96}\left(\theta \gamma_{[m n}{ }^{q} \theta\right)\left(\theta \gamma_{p] r s} \theta\right) \partial_{q} F_{r s}-\frac{7}{96}\left(\theta \gamma_{[m n \mid t} \theta\right)\left(\theta \gamma^{r s t} \theta\right) \partial_{\mid p]} F_{r s}
\end{aligned}
$$

The first two lines result directly from applying the supercovariant derivative $D_{\alpha}=\partial_{\alpha}+$ $\frac{1}{2}(\theta \not \partial)_{\alpha}$ to the lowest terms in the expansion of $A_{\alpha}$, followed by simple gamma algebra. For the third line, we note that the $S O(10)$ representation content of $\theta^{3}$ and of $\partial_{m} \chi^{\alpha}$ predicts two independent three-forms composed of these objects. Indeed, writing $\theta^{3}$ as a $\gamma$-traceless spinor-two-form $\Theta_{\alpha}^{a b}$ via $\Theta_{\alpha}^{a b}=\left(\theta \gamma^{a b c} \theta\right)\left(\gamma_{c} \theta\right)_{\alpha}$, which captures the whole content of $\theta^{3}$ because $\left(\theta \gamma^{a b c} \theta\right) \theta^{\alpha}=\frac{1}{2}\left(\gamma^{[a} \Theta^{b c]}\right)^{\alpha}$, we can see that those two independent three-forms are given by $\left(\Theta_{[m n} \partial_{p]} \chi\right)$ and $\left(\Theta_{a[m} \gamma_{n p]} \partial^{a} \chi\right)$. The easiest way to obtain their coefficients is then to go to a gamma matrix representation. Similarly, for the fourth line, there are two independent three-forms indicated by representation content, and one can use the Bianchi identity $\partial_{[a} F_{b c]}=0$ as well as the spinor product identities $\left(\gamma_{a b} \theta\right)_{\alpha}\left(\theta \gamma^{a b c} \theta\right)=0$, $\left(\theta \gamma_{a}{ }^{[m n} \theta\right)\left(\theta \gamma^{p] q a} \theta\right)=0$ and $\left(\theta \gamma^{m n p q r}{ }_{a b} \theta\right)\left(\theta^{s a b} \theta\right)=20\left(\theta \gamma^{[m n p} \theta\right)\left(\theta \gamma^{q r] s} \theta\right)$ to reduce all terms in $\left(D \gamma_{m n p} A\right)^{(4)}$ to the form given on the right-hand side.

\section{D.2. Correlator catalog}

The most efficient way to evaluate pure spinor superspace correlators is to compile a "catalog" of building blocks, as outlined in appendix A of [22]. This is particularly the case if one completely automates the process that deconstructs an arbitrary correlator into these blocks, namely by expanding gamma products, sorting of spinor bilinears and, for fermionic fields, applying Fierz rearrangements. Two different approaches, the automatic 
conversion to traces and the component-based evaluation studied in [19, have turned out much slower and have not been used here 19 .

In principle the three building blocks $\left\langle\left(\lambda \gamma^{[5]} \lambda\right)\left(\lambda \gamma^{[1,3,5]} \theta\right)\left(\theta \gamma^{[3]} \theta\right)\left(\theta \gamma^{[3]} \theta\right)\right\rangle$ are sufficient, but it is more efficient to also allow for the pairings $\langle(\lambda \gamma \theta)(\lambda \gamma \theta)(\lambda \gamma \theta)(\theta \gamma \theta)\rangle$, since they only need to be computed once, making all subsequent computations faster. In addition to the identities listed in [22] and eq. (2.7) of [19], we use the following:

$$
\begin{aligned}
& \left\langle\left(\lambda \gamma^{a_{1} \ldots a_{5}} \theta\right)\left(\lambda \gamma^{b_{1} b_{2} b_{3}} \theta\right)\left(\lambda \gamma^{c_{1} c_{2} c_{3}} \theta\right)\left(\theta \gamma^{d_{1} d_{2} d_{3}} \theta\right)\right\rangle= \\
& -\frac{3}{35}\left(\delta_{e_{1} \ldots e_{5}}^{a_{1} \ldots a_{5}}+\frac{1}{5 !} \epsilon^{a_{1} \ldots a_{5}} e_{1} \ldots e_{5}\right)\left[\left(\delta_{b_{1} b_{2} b_{3} c_{1} d_{1}}^{e_{1} e_{2} e_{3} e_{4} e_{5}} \delta_{c_{2} c_{3}}^{d_{2} d_{3}}-\delta_{c_{1} c_{2} c_{3} b_{1} d_{1}}^{e_{1} e_{2} e_{3} e_{4} e_{5}} \delta_{b_{2} b_{3}}^{d_{2} d_{3}}\right)\right. \\
& \left.+\delta_{b_{1} b_{2} c_{1} c_{2} d_{1}}^{e_{1} e_{2} e_{3} e_{4} e_{5}} \delta_{b_{3} c_{3}}^{d_{2} d_{3}}+\left(\delta_{b_{1} b_{2} c_{1} d_{1} d_{2}}^{e_{1} e_{2} e_{3} e_{4} e_{5}} \delta_{c_{2} c_{3}}^{b_{3} d_{3}}-\delta_{c_{1} c_{2} b_{1} d_{1} d_{2}}^{e_{1} e_{2} e_{3} e_{4} e_{5}} \delta_{b_{2} b_{3}}^{c_{3} d_{3}}\right)-\delta_{b_{1} c_{1} d_{1} d_{2} d_{3}}^{e_{1} e_{2} e_{3} e_{4} e_{5}} \delta_{c_{2} c_{3}}^{b_{2} b_{3}}\right]_{\left[b_{i}\right]\left[c_{i}\right]\left[d_{i}\right]} \\
& \left\langle\left(\lambda \gamma^{a_{1} \ldots a_{5}} \theta\right)\left(\lambda \gamma^{b_{1} \ldots b_{5}} \theta\right)\left(\lambda \gamma^{c} \theta\right)\left(\theta \gamma^{d_{1} d_{2} d_{3}} \theta\right)\right\rangle=-\frac{1}{7}\left(\delta_{e_{1} \ldots e_{5}}^{a_{1} \ldots a_{5}}+\frac{1}{5 !} \epsilon^{a_{1} \ldots a_{5}} e_{1} \ldots e_{5}\right) \\
& \times\left[\delta_{b_{1} b_{2} b_{3} b_{4} d_{1}}^{e_{1} e_{2} e_{3} e_{4} e_{5}} \delta_{b_{5}}^{d_{2}} \delta_{d_{3}}^{c}-2 \delta_{b_{1} b_{2} b_{3}}^{e_{1} e_{2} e_{3}}\left(\delta_{c d_{1}}^{e_{4} e_{5}} \delta_{b_{4} b_{5}}^{d_{2} d_{3}}-\delta_{d_{2} d_{3}}^{e_{4} e_{5}} \delta_{b_{4} b_{5}}^{c d_{1}}\right)\right]_{\left[b_{i}\right]\left[d_{i}\right]} \\
& \left\langle\left(\lambda \gamma^{a_{1} \ldots a_{5}} \theta\right)\left(\lambda \gamma^{b_{1} \ldots b_{5}} \theta\right)\left(\lambda \gamma^{c_{1} c_{2} c_{3}} \theta\right)\left(\theta \gamma^{d_{1} d_{2} d_{3}} \theta\right)\right\rangle=\frac{2}{7}\left(\delta_{e_{1} \ldots e_{5}}^{a_{1} \ldots a_{5}}+\frac{1}{5 !} \epsilon^{a_{1} \ldots a_{5}} e_{1} \ldots e_{5}\right) \\
& \left.\times\left[\delta_{b_{1} b_{2} b_{3} b_{4}}^{e_{1} e_{2} e_{3} e_{4}}\left(\delta_{c_{1}}^{e_{5}} \delta_{b_{5}}^{d_{1}}-\delta_{d_{1}}^{e_{5}} \delta_{b_{5}}^{c_{1}}\right) \delta_{d_{2} d_{3}}^{c_{2} c_{3}}-2 \delta_{b_{1} b_{2} b_{3}}^{e_{1} e_{2} e_{3}}\left(\delta_{c_{1} c_{2}}^{e_{4} e_{5}} \delta_{b_{4} b_{5}}^{d_{1} d_{2}}-\delta_{d_{1} d_{2}}^{e_{4} e_{5}} \delta_{b_{4} b_{5}}^{c_{1} c_{2}}\right) \delta_{d_{3}}^{c_{3}}\right)\right]_{\left[b_{i}\right]\left[c_{i}\right]\left[d_{i}\right]}(\mathrm{D}
\end{aligned}
$$

They have been derived using a symmetry-based ansatz, as described in [17]. A useful application of the component-based method of [19] is that it provides a quick way to compute the coefficients in such an ansatz.

\section{D.3. Kinematic reduction}

In the component calculations relevant to this paper, we encounter kinematic factors that are Lorentz invariant polynomials in the momenta $k_{\mu}^{i}$ as well as the polarization vectors $e_{\mu}^{i}$ and/or the fermionic spinor wavefunctions $\chi^{i \alpha}$. Due to the on-shell identities $\sum_{i} k^{i}=\left(k^{i}\right)^{2}=k^{i} \cdot e^{i}=\not k^{i} \chi^{i}=0$, there are many relations among these polynomials, and

19 Trace evaluations using Mathematica / GAMMA become very slow once the number of gamma matrices in the trace reaches the mid-twenties. Calculations of traces that take (tens of) hours with Mathematica typically finish within (tens of) minutes using FORM. Still, FORM takes several hours to compute an expression like (C.9) starting from the spinorial formula (C.2). On the other hand, using the "catalog method", the correlator evaluation becomes a matter of seconds. A difference in performance of about an order of magnitude remains in favor of FORM, due to the handling of antisymmetric tensors and of dummy indices in the GAMMA package. 
we need to be able to systematically reduce them to some sets of independent kinematic invariants.

As will be shown in the following, such reduction algorithms can be found in the form of a collection of replacement rules, which can easily be implemented on a computer algebra system. An alternative method considered in [19] was based on evaluating symbolic expressions on a number of integer-valued solutions to the on-shell identities. As the number of external fields and therefore the number of independent kinematic structures increases, this method becomes unpractical and slow. However, the component-based method is still useful to ensure that the end products of the replacement rules are in fact linearly independent, that is, that no identities have been missed.

For bosonic expressions, the reduction process is very simple and consists of eliminating one momentum (say $k^{5} \rightarrow-k^{1}-\cdots-k^{4}$ ) in all $k \cdot e$ and $k \cdot k$ products, plus one additional product like $k^{4} \cdot e^{5} \rightarrow-\left(k^{1}+k^{2}+k^{3}\right) \cdot e^{5}$, and one $k \cdot k$ combination to resolve the relation $\frac{1}{2}\left(\sum_{i=1}^{4} k^{i}\right)^{2}=\sum_{i<j}^{4} k^{i} \cdot k^{j}=0$. This leaves five independent quadratic momentum invariants, and three scalar products for every polarization. Note that any terms containing the ten-index epsilon tensor $\epsilon_{10}$ will vanish, since there are only five polarization vectors, and the five momenta are linearly dependent.

Adding in two fermions only requires a mild generalization, as they can be re-written into independent antisymmetric tensors $\left(\chi^{1} \gamma^{[k]} \chi^{2}\right)$. Any $\epsilon_{10}$ terms can be eliminated by dualizing $\epsilon_{10} \gamma^{[k]} \rightarrow \gamma^{[10-k]}$, since the $\chi^{i}$ are chiral spinors. The only complication arises from relations due to the Dirac equations $\not k^{1} \chi^{1}=\not k^{2} \chi^{2}=0$, but these can be resolved by replacing

$$
\left(\chi^{1} \gamma^{a_{1} \ldots a_{n}} \chi^{2}\right) k_{a_{1}}^{1} \rightarrow-(n-1) k^{1\left[a_{2}\right.}\left(\chi^{1} \gamma^{\left.a_{3} \ldots a_{n}\right]} \chi^{2}\right)
$$

and similarly for $k^{2}$. This rule, along with the dualization step, allows us to regard the spinor bilinears as unconstrained antisymmetric tensors from now on. After applying the bosonic simplification rules and resolving the tensor antisymmetry by sorting into some order, e.g. $k_{a_{1}}^{2} e_{a_{2}}^{5} k_{a_{3}}^{1} \gamma^{a_{1} a_{2} a_{3} \cdots} \rightarrow e_{a_{1}}^{5} k_{a_{2}}^{1} k_{a_{3}}^{2} \gamma^{a_{1} a_{2} a_{3} \ldots}$ etc., all remaining structures are independent. In the five-point amplitude calculation, where we are dealing with terms of the form $k k k k \chi^{1} \chi^{2} e^{3} e^{4} e^{5}$, we find 476 independent kinematic structures.

With four fermions, we have to think about Fierz transformations which might seem to lead to relations that are hard to resolve algorithmically. Fortunately, since we are dealing with distinct spinors, we can completely avoid this issue by rearranging all spinor products into the same order, for example into $\left(\chi^{1} \gamma^{[k]} \chi^{2}\right)\left(\chi^{3} \gamma^{[l]} \chi^{4}\right)$ bilinears, at the outset 
of the reduction procedure. We then eliminate $\gamma^{[k]} \rightarrow \epsilon_{10} \gamma^{[10-k]}$ whenever $k>5$, re-write $\epsilon_{10} \epsilon_{10} \rightarrow \delta$, dualize $\epsilon_{10} \gamma^{[5]} \rightarrow \gamma^{[5]}$, and apply the Dirac equation replacement (D.5) as well as the bosonic simplification rules.

The resulting terms will not all be independent, since the duality properties of the spinor bilinears have not been dealt with, and this is slightly more involved than in the two-fermion case. For example, the contraction of two self-dual five-forms vanishes, so all terms containing $\left(\chi^{1} \gamma^{a_{1} \ldots a_{5}} \chi^{2}\right)\left(\chi^{3} \gamma_{a_{1} \ldots a_{5}} \chi^{4}\right)$ have to be set to zero. We now show how to resolve this issue for expressions of the form $\chi^{1} \chi^{2} \chi^{3} \chi^{4} e^{5} k k k$ as encountered in the fivepoint amplitude. The steps described in the last paragraph lead to the following structures: First, there are 30 possible terms in which the spinors combine into a scalar,

$$
\left(\left(\chi^{1} \gamma^{a_{1}} \chi^{2}\right)\left(\chi^{3} \gamma_{a_{1}} \chi^{4}\right) \text { or }\left(\chi^{1} \gamma^{a_{1} a_{2} a_{3}} \chi^{2}\right)\left(\chi^{3} \gamma_{a_{1} a_{2} a_{3}} \chi^{4}\right)\right) \times(k \cdot k)\left(e^{5} \cdot k^{1 / 2 / 3}\right)
$$

where we have already set to zero the $\gamma^{[5]} \cdot \gamma^{[5]}$ products. Next, there are 116 two-tensor combinations,

$$
\begin{aligned}
& \left(\left(\chi^{1} \gamma^{m_{1}} \chi^{2}\right)\left(\chi^{3} \gamma^{m_{2}} \chi^{4}\right) \text { or }\left(\chi^{1} \gamma^{a_{1} a_{2} m_{1}} \chi^{2}\right)\left(\chi^{3} \gamma_{a_{1} a_{2}}^{m_{2}} \chi^{4}\right)\right) \\
& \times\left(k_{m_{1}}^{3 / 4} k_{m_{2}}^{1 / 2}\left(e^{5} \cdot k^{1 / 2 / 3}\right) \text { or } k_{m_{1}}^{3 / 4} e_{m_{2}}^{5}(k \cdot k) \text { or } e_{m_{1}}^{5} k_{m_{2}}^{1 / 2}(k \cdot k)\right), \\
& \left(\left(\chi^{1} \gamma^{a_{1} m_{1} m_{2}} \chi^{2}\right)\left(\chi^{3} \gamma_{a_{1}} \chi^{4}\right) \text { or }\left(\chi^{1} \gamma^{a_{1} a_{2} a_{3} m_{1} m_{2}} \chi^{2}\right)\left(\chi^{3} \gamma_{a_{1} a_{2} a_{3}} \chi_{4}\right)\right) \\
& \times\left(k_{m_{1}}^{3} k_{m_{2}}^{4}\left(e^{5} \cdot k^{1 / 2 / 3}\right) \text { or } k_{m_{1}}^{3 / 4} e_{m_{2}}^{5}(k \cdot k)\right), \\
& \left(\left(\chi^{1} \gamma^{a_{1}} \chi^{2}\right)\left(\chi^{3} \gamma_{a_{1}}^{m_{1} m_{2}} \chi^{4}\right) \text { or }\left(\chi^{1} \gamma^{a_{1} a_{2} a_{3}} \chi^{2}\right)\left(\chi^{3} \gamma_{a_{1} a_{2} a_{3}}^{m_{1} m_{2}} \chi^{4}\right)\right) \\
& \times\left(k_{m_{1}}^{1} k_{m_{2}}^{2}\left(e^{5} \cdot k^{1 / 2 / 3}\right) \text { or } k_{m_{1}}^{1 / 2} e_{m_{2}}^{5}(k \cdot k)\right) .
\end{aligned}
$$

Finally, there are 8 four-tensor combinations,

$$
\begin{aligned}
& \left(\chi^{1} \gamma^{m_{1} m_{2} m_{3}} \chi^{2}\right)\left(\chi^{3} \gamma^{m_{4}} \chi^{4}\right) \times k_{m_{1}}^{3} k_{m_{2}}^{4} e_{m_{3}}^{5} k_{m_{4}}^{1 / 2}, \\
& \left(\chi^{1} \gamma^{m_{1}} \chi^{2}\right)\left(\chi^{3} \gamma^{m_{2} m_{3} m_{4}} \chi^{4}\right) \times k_{m_{1}}^{3 / 4} k_{m_{2}}^{1} k_{m_{3}}^{2} e_{m_{4}}^{5}, \\
& \left(\chi^{1} \gamma^{a_{1} m_{1} m_{2}} \chi^{2}\right)\left(\chi^{3} \gamma_{a_{1}}^{m_{3} m_{4}} \chi^{4}\right) \times\left(k_{m_{1}}^{3} k_{m_{2}}^{4} e_{m_{3}}^{5} k_{m_{4}}^{1 / 2} \text { or } k_{m_{1}}^{3} k_{m_{2}}^{4} e_{m_{3}}^{5} k_{m_{4}}^{1 / 2}\right) .
\end{aligned}
$$

These 154 structures are independent, as can be seen by going to components, and it will turn out that they form a complete kinematic set. There are three more groups of possible outcomes, but they can be simplified using duality manipulations. The first group contains a four-tensor of the form $\epsilon_{10}\left(\chi^{1} \gamma^{[3]} \chi^{2}\right)\left(\chi^{3} \gamma^{[3]} \chi^{4}\right)$ contracted into $k k k e^{5}$ :

$$
X_{1}=\epsilon^{m_{1} \ldots m_{4}} a_{1} a_{2} a_{3} b_{1} b_{2} b_{3}\left(\chi^{1} \gamma^{a_{1} a_{2} a_{3}} \chi^{2}\right)\left(\chi^{3} \gamma^{b_{1} b_{2} b_{3}} \chi^{4}\right) \times k_{m_{1}}^{1} k_{m_{2}}^{2} k_{m_{3}}^{3} e_{m_{4}}^{5}
$$


and three others with $k^{1} k^{2} k^{4}, k^{1} k^{3} k^{4}$, or $k^{2} k^{3} k^{4}$. Upon dualising $\epsilon \gamma^{3} \rightarrow \gamma^{7}$,

$$
X_{1}=3 !\left(\chi^{1} \gamma_{m_{1} m_{2} m_{3} m_{4} b_{1} b_{2} b_{3} b_{4}} \chi^{2}\right)\left(\chi^{3} \gamma^{b_{1} b_{2} b_{3}} \chi^{4}\right) \times k_{m_{1}}^{1} k_{m_{2}}^{2} k_{m_{3}}^{3}
$$

the momenta $k^{1}$ and $k^{2}$ are contracted into the $\left(\chi^{1} \chi^{2}\right)$ bilinear and the Dirac equation can be used to reduce $\gamma^{[7]} \rightarrow \gamma^{[5]}$ and thereby relate $X_{1}$ to the previous list. The second group consists of

$$
\begin{aligned}
& Y_{1 / 2}=\left(\chi^{1} \gamma^{a_{1} a_{2} m_{1} m_{2} m_{3}} \chi^{2}\right)\left(\chi^{3} \gamma_{a_{1} a_{2}}^{m_{4}} \chi^{4}\right) \times k_{m_{1}}^{3} k_{m_{2}}^{4} e_{m_{3}}^{5} k_{m_{4}}^{1 / 2} \\
& Y_{3 / 4}=\left(\chi^{1} \gamma^{a_{1} a_{2} m_{1}} \chi^{2}\right)\left(\chi^{3} \gamma_{a_{1} a_{2}}^{m_{2} m_{3} m_{4}} \chi^{4}\right) \times k_{m_{1}}^{3 / 4} k_{m_{2}}^{1} k_{m_{3}}^{2} e_{m_{4}}^{5}
\end{aligned}
$$

and can be reduced similarly. For example, in the first line, dualization leads to

$$
\left(\frac{1}{20} \delta_{m_{1} m_{2} m_{3}}^{d_{1} d_{2} m_{4}}\left(\chi^{1} \gamma^{d_{3} \ldots d_{7}} \chi^{2}\right)-\frac{1}{12} \delta_{m_{1} m_{2} m_{3}}^{d_{1} d_{2} d_{3}}\left(\chi^{1} \gamma^{d_{4} \ldots d_{7} m_{4}} \chi^{2}\right)\right)\left(\chi^{3} \gamma_{d_{1} \ldots d_{7}} \chi^{4}\right) \times k_{m_{1}}^{3} k_{m_{2}}^{4} e_{m_{3}}^{5} k_{m_{4}}^{1 / 2}
$$

and now at least one momentum is contracted with a bilinear where the Dirac equation can be used. The third group contains two $\gamma^{[5]}$ factors, either as two-tensor

$$
Z^{m_{1} m_{2}}=\left(\chi^{1} \gamma^{a_{1} a_{2} a_{3} a_{4} m_{1}} \chi^{2}\right)\left(\chi^{3} \gamma_{a_{1} a_{2} a_{3} a_{4}}^{m_{2}} \chi^{4}\right)
$$

contracted into at least one momentum, or as four-tensor

$$
Z^{m_{1} m_{2}, m_{3} m_{4}}=\left(\chi^{1} \gamma^{a_{1} a_{2} a_{3} m_{1} m_{2}} \chi^{2}\right)\left(\chi^{3} \gamma_{a_{1} a_{2} a_{3}}^{m_{3} m_{4}} \chi^{4}\right)
$$

contracted into three momenta and the polarization $e^{5}$. Here we note that the only twotensor irrep contained in the tensor product of two self-dual five-forms is the symmetric traceless one, so we must have $Z^{\left[m_{1} m_{2}\right]}=0$. Similarly, the product does not contain a completely antisymmetric four-tensor, and hence $Z^{\left[m_{1} m_{2}, m_{3} m_{4}\right]}=0$, which implies

$Z^{m_{1} m_{2}, m_{3} m_{4}}=Z^{m_{1} m_{3}, m_{2} m_{4}}-Z^{m_{1} m_{4}, m_{2} m_{3}}-Z^{m_{2} m_{3}, m_{1} m_{4}}+Z^{m_{2} m_{4}, m_{1} m_{3}}-Z^{m_{3} m_{4}, m_{1} m_{2}}$.

In all cases, the symmetries of $Z$ allow us to shuffle at least one momentum onto a bilinear where the Dirac equation can be used, relating all terms containing $Z$ tensors to the list of 154 independent structures. This concludes the simplification of four-fermion terms. 


\section{References}

[1] D. J. Gross and J. H. Sloan, "The Quartic Effective Action for the Heterotic String," Nucl. Phys. B 291, 41 (1987).

[2] D. J. Gross and E. Witten, "Superstring Modifications Of Einstein's Equations," Nucl. Phys. B 277, 1 (1986).

[3] P. Ramond, "Dual Theory for Free Fermions," Phys. Rev. D 3, 2415 (1971) ;

A. Neveu and J. H. Schwarz, "Factorizable dual model of pions," Nucl. Phys. B 31 (1971) 86 ;

A. Neveu and J. H. Schwarz, "Quark Model of Dual Pions," Phys. Rev. D 4, 1109 (1971).

[4] M. B. Green and J. H. Schwarz, "Covariant Description Of Superstrings," Phys. Lett. B 136, 367 (1984);

M. B. Green and J. H. Schwarz, "Supersymmetrical String Theories," Phys. Lett. B 109, 444 (1982).

[5] J. H. Schwarz, "Superstring Theory," Phys. Rept. 89, 223 (1982).

[6] E. D'Hoker and D. H. Phong, "Two-Loop Superstrings VI: Non-Renormalization Theorems and the 4-Point Function," Nucl. Phys. B 715, 3 (2005) arXiv:hep-th/0501197

[7] K. Peeters, P. Vanhove and A. Westerberg, "Chiral splitting and world-sheet gravitinos in higher-derivative string amplitudes," Class. Quant. Grav. 19, 2699 (2002) arXiv:hep-th/0112157;

K. Peeters, P. Vanhove and A. Westerberg, "Supersymmetric higher-derivative actions in ten and eleven dimensions, the associated superalgebras and their formulation in superspace," Class. Quant. Grav. 18, 843 (2001) arXiv:hep-th/0010167.

[8] N. Berkovits, "Super-Poincare covariant quantization of the superstring," JHEP 0004, 018 (2000) arXiv:hep-th/0001035.

[9] N. Berkovits, "ICTP lectures on covariant quantization of the superstring," arXiv:hepth/0209059.

[10] N. Berkovits and B. C. Vallilo, "Consistency of super-Poincare covariant superstring tree amplitudes," JHEP 0007, 015 (2000) arXiv:hep-th/0004171.

[11] N. Berkovits, "New higher-derivative R**4 theorems," Phys. Rev. Lett. 98, 211601 (2007) arXiv:hep-th/0609006.

[12] A. Basu, "The $\mathrm{D}^{* *} 10 \mathrm{R}^{* *} 4$ term in type IIB string theory," Phys. Lett. B 648, 378 (2007) arXiv:hep-th/0610335.

[13] M. B. Green, J. G. Russo and P. Vanhove, "Ultraviolet properties of maximal supergravity," Phys. Rev. Lett. 98, 131602 (2007) arXiv:hep-th/0611273.

[14] N. Berkovits, "Multiloop amplitudes and vanishing theorems using the pure spinor formalism for the superstring," JHEP 0409, 047 (2004) [arXiv:hep-th/0406055]. 
[15] N. Berkovits, "Super-Poincare covariant two-loop superstring amplitudes," JHEP 0601, 005 (2006) arXiv:hep-th/0503197.

[16] N. Berkovits, "Explaining pure spinor superspace," arXiv:hep-th/0612021.

[17] N. Berkovits and C. R. Mafra, "Equivalence of two-loop superstring amplitudes in the pure spinor and RNS formalisms," Phys. Rev. Lett. 96, 011602 (2006) arXiv:hepth/0509234.

[18] C. R. Mafra, "Four-point one-loop amplitude computation in the pure spinor formalism," JHEP 0601, 075 (2006) arXiv:hep-th/0512052].

[19] C. Stahn, "Fermionic superstring loop amplitudes in the pure spinor formalism," JHEP 0705, 034 (2007) [arXiv:0704.0015 [hep-th]].

[20] C. R. Mafra, "Pure Spinor Superspace Identities for Massless Four-point Kinematic Factors," JHEP 0804, 093 (2008) arXiv:0801.0580 [hep-th]].

[21] N. Berkovits, "Pure spinor formalism as an $\mathrm{N}=2$ topological string," JHEP 0510, 089 (2005) arXiv:hep-th/0509120.

[22] N. Berkovits and C. R. Mafra, "Some superstring amplitude computations with the non-minimal pure spinor formalism," JHEP 0611, 079 (2006) arXiv:hep-th/0607187.

[23] J. Hoogeveen and K. Skenderis, "BRST quantization of the pure spinor superstring," JHEP 0711, 081 (2007) arXiv:0710.2598 [hep-th]].

[24] N. Berkovits and N. Nekrasov, "Multiloop superstring amplitudes from non-minimal pure spinor formalism," JHEP 0612, 029 (2006) [arXiv:hep-th/0609012].

[25] G. Policastro and D. Tsimpis, "R**4, purified," arXiv:hep-th/0603165.

[26] Z. H. Lin, "One Loop Closed String Five Particle Fermion Amplitudes in the Covariant Formulation," Int. J. Mod. Phys. A 5, 299 (1990) ;

Z. H. Lin, L. Clavelli and S. T. Jones, "Five Point Function in the Covariant Formulation of the Type I Superstring Theory," Nucl. Phys. B 294, 83 (1987).

[27] J. J. Atick and A. Sen, "Covariant one loop fermion emission amplitudes in closed string theories," Nucl. Phys. B 293, 317 (1987).

[28] K. Lee and W. Siegel, "Simpler superstring scattering," JHEP 0606, 046 (2006) arXiv:hep-th/0603218.

[29] D. M. Richards, "The One-Loop Five-Graviton Amplitude and the Effective Action," JHEP 0810, 042 (2008) [arXiv:0807.2421 [hep-th]].

[30] P. H. Frampton, P. Moxhay and Y. J. Ng, "Explicit Evaluation Of Pentagon Diagram For Open Superstrings," Nucl. Phys. B 276, 599 (1986).

[31] A. Tsuchiya, "More on One Loop Massless Amplitudes of Superstring Theories," Phys. Rev. D 39, 1626 (1989).

[32] C. R. Mafra, "Superstring Scattering Amplitudes with the Pure Spinor Formalism", Ph.D thesis, September 2008, arXiv:0902.1552 [hep-th]. 
[33] J. A. M. Vermaseren, "New features of FORM," arXiv:math-ph/0010025 ;

M. Tentyukov and J. A. M. Vermaseren, "The multithreaded version of FORM," arXiv:hep-ph/0702279.

[34] C. R. Mafra, "PSS: A FORM Program to Compute Pure Spinor Superspace Expressions", unpublished.

[35] U. Gran, "GAMMA: A Mathematica package for performing Gamma-matrix algebra and Fierz transformations in arbitrary dimensions," arXiv:hep-th/0105086.

[36] E. P. Verlinde and H. L. Verlinde, "Chiral bosonization, determinants and the string partition function," Nucl. Phys. B 288, 357 (1987).

[37] J. Polchinski, "String theory. Vol. 1: An Introduction to the Bosonic String," Cambridge, UK: Univ. Pr. (1998) 402 $p$

[38] M. B. Green, J. H. Schwarz and E. Witten, "Superstring Theory. Vol. 2: Loop Amplitudes, Anomalies And Phenomenology," Cambridge, UK: Univ. Pr. (1987) 596 p. (Cambridge Monographs On Mathematical Physics)

[39] L. A. Barreiro and R. Medina, "5-field terms in the open superstring effective action," JHEP 0503, 055 (2005) arXiv:hep-th/0503182.

[40] R. Medina, F. T. Brandt and F. R. Machado, "The open superstring 5-point amplitude revisited," JHEP 0207, 071 (2002) arXiv:hep-th/0208121.

[41] S. Stieberger and T. R. Taylor, "Non-Abelian Born-Infeld action and type I - heterotic duality. II: Nonrenormalization theorems," Nucl. Phys. B 648, 3 (2003) arXiv:hepth/0209064.

[42] A. M. Cohen, M. van Leeuwen and B. Lisser, LiE v.2.2.2, http://www-math.univpoitiers.fr/ maavl/LiE/

[43] J. P. Harnad and S. Shnider, "Constraints And Field Equations For Ten-Dimensional Superyang-Mills Theory," Commun. Math. Phys. 106 (1986) 183 ;

H. Ooguri, J. Rahmfeld, H. Robins and J. Tannenhauser, "Holography in superspace," JHEP 0007, 045 (2000) arXiv:hep-th/0007104 ;

P. A. Grassi and L. Tamassia, "Vertex operators for closed superstrings," JHEP 0407, 071 (2004) arXiv:hep-th/0405072. 